\title{
BIRATIONAL CLASSIFICATION OF CURVES ON RATIONAL SURFACES
}

\author{
ALBERTO CALABRI AND CIRO CILIBERTO
}

\begin{abstract}
In this paper we consider the birational classification of pairs $(S, \mathcal{L})$, with $S$ a rational surface and $\mathcal{L}$ a linear system on $S$. We give a classification theorem for such pairs, and we determine, for each irreducible plane curve $B$, its Cremona minimal models, that is, those plane curves which are equivalent to $B$ via a Cremona transformation and have minimal degree under this condition.
\end{abstract}

\section{Introduction}

Let $B$ be an irreducible curve in the complex projective plane $\mathbb{P}^{2}$. A natural question is to ask about its Cremona minimal models, that is, for those curves which are equivalent to $B$ via a Cremona transformation of $\mathbb{P}^{2}$ and have minimal degree under this condition. More generally, the same question can be asked for positive dimensional linear systems $\mathcal{L}$ of plane curves.

This is a classical problem, which goes back to the very beginnings of birational geometry in the second half of the nineteenth century, the main characters being Cremona and his school and Noether. This question was later considered by several algebraic geometers for many decades until the 1940s. Indeed, a long series of papers by various classical authors was devoted to trying to solve this problem at least for planar linear systems of curves of low genera. Giving here a full account, with complete references, of all these attempts, some of them affected by serious gaps, would turn these few lines into a historical work rather than an introduction. So we direct the interested reader to Coolidge's book [Coo], whose first edition appeared in 1931, which contains an exposition of the classical results and a detailed bibliography on the subject. Another beautiful classical reference is the book [Con], which contains the most advanced classical treatment of the subject, as well as interesting historical notes. We cannot resist, however, mention-

\footnotetext{
Received June 30, 2009. Accepted October 29, 2009.

2000 Mathematics Subject Classification. Primary 14E05; Secondary 14J26, 14H50, 14E07, 14E30.
}

(C) 2010 by The Editorial Board of the Nagoya Mathematical Journal 
ing, among all the others, Castelnuovo's contributions ([Ca1], [Ca2], [Ca3]; see also $[\mathrm{K} 1],[\mathrm{K} 2],[\mathrm{No}])$, where adjunction theory is fully exploited in a form that, after Mori's epochal work, we call "running a minimal model program" driven by a given divisor on a surface, that is, a $\sharp$-minimal model program in M. Reid's terminology [R].

In modern times, the question has been considered again starting from the 1960s by a few authors, among which, in chronological order, we mention Nagata ([Na1], [Na2]), Kumar and Murthy [KM], Reid [R], Dicks [D], Iitaka ([I1], [I2]), and Matsuda [M].

In a nutshell, the problem boils down to considering the birational classification of pairs $(S, \mathcal{L})$, with $S$ a rational surface and $\mathcal{L}$ a linear system on it. Taking this viewpoint, the most appropriate tool available today for attacking the question consists in using the machinery of Mori's program, in its $\log$ version. This is essentially Dick's [D] and Reid's [R] viewpoint, and this is basically what we also do here, though we use Iitaka's terminology ([I1], [I2]) and the more classical approach via adjunction theory á la Castelnuovo. Part of our main results is stated in the following two theorems (for more detailed statements, see Theorems 9.4 and 9.7).

TheOREm 0.1. Let $(S, C)$ be a pair, with $S$ rational and $C$ smooth and irreducible, which is not birationally equivalent to $\left(\mathbb{P}^{2}, L\right)$, where $L$ is a line. Let $m$ be the maximum integer such that $\left|C+m K_{S}\right|$ is not empty, and let $\alpha$ be the dimension of this system. Then $(S, C)$ is birationally equivalent to one of the following pairs.

(i) $\left(\mathbb{P}^{2}, D\right)$, where $D$ is a plane curve of degree $d \geqslant 3$ with points of multiplicity strictly smaller than $m-1$, where $m=[d / 3]$.

(ii) $\left(\mathbb{F}_{n}, D\right)$, where $\mathbb{F}_{n}=\mathbb{P}\left(\mathcal{O}_{\mathbb{P}^{1}} \oplus \mathcal{O}_{\mathbb{P}^{1}}(-n)\right)$;

$$
D \in\left|(2 m+\epsilon) E+\left((2+n) m+\frac{\alpha+\epsilon(n-1)}{1+\epsilon}\right) F\right|,
$$

with $\epsilon \in\{0,1\}, E$ a curve with $E^{2}=-n$, and $F$ a ruling of $\mathbb{F}_{n}$; and

- $D$ is irreducible with points of multiplicity at most $m$, and

- if $\epsilon=0$ and $n>0$, the singular points of $D$ of multiplicity $m$ lie on $E$ and $n$ is minimal under this condition.

These pairs may be birationally equivalent only if

- $n=\epsilon=0$ and $D$ has at least two points of multiplicity m; and

- $n=\epsilon=1, \alpha=0$ (resp., $\alpha=2$ ), and $D$ has at least three (resp., two) points of multiplicity $m$. 
Theorem 0.2. Let $C$ be an irreducible plane curve. Then a Cremona minimal model $B$ of $C$ is one of the following types:

(i) $B$ is a line;

(ii) if $B$ has degree $d$ and points of multiplicities $m_{1}, \ldots, m_{r}$ with $m_{1} \geqslant$ $\cdots \geqslant m_{r}$, then $d \geqslant m_{1}+m_{2}+m_{3}$;

(iii) $B$ is a curve of degree $d$ with a point $p$ of maximal multiplicity $m_{0}$, and all points of multiplicity $\mu \geqslant\left(d-m_{0}\right) / 2$ are infinitely near to $p$.

The structure of the Cremona minimal curves in (iii) can be well specified, as in the statement of Theorem 9.7. They are obtained from the pairs $\left(\mathbb{F}_{n}, D\right)$ in Theorem 0.1 with a suitable process which is explained in detail in Section 8. The curves in (ii) may be birationally, and not projectively, equivalent only if $d=m_{1}+m_{2}+m_{3}$. This is a consequence of a result, asserted by several classical authors and proved by G. Jung [J], to the effect that a linear system of plane curves of degree $d$ with points of multiplicities $m_{1} \geqslant m_{2} \geqslant m_{3} \geqslant \cdots$ is Cremona minimal as soon as $d \geqslant m_{1}+m_{2}+m_{3}$, and if $d>m_{1}+m_{2}+m_{3}$, any Cremona minimal linear system birational to it is projectively equivalent to it. We give a short and easy proof of this in Section 2. As for type (iii), it is possible to have several different Cremona minimal, nonprojectively equivalent models, with different multiplicities.

In our view, these results completely solve the classification problem, though the difficulty remains, given a specific curve $B$, of resolving its singularities and determining the structure of its subsequent adjoint linear systems.

The paper is organized as follows. In Section 1 we fix notation and recall a few facts about infinitely near points and linear systems. In Section 2 we prove some basic results on Cremona minimality and the aforementioned theorem of Jung. In Section 3 we recall a few properties of $(-1)$-cycles, that is, those effective divisors $C$ on a smooth surface $S$ which are contracted to a smooth point by a birational morphism, which is an isomorphism on the complement of $C$ on $S$. This is used in Section 4, where we prove some essential nefness results on effective adjoint linear systems which boil down to computing their Zariski decomposition, and we recall how adjoint systems behave under birational maps. In Section 5 we introduce Iitaka's $\sharp$-models and define the equally useful $b$-models and $\downarrow$-models. We devote Section 6 to briefly recalling the birational classification of pairs $(S, \mathcal{L})$, with $\mathcal{L}$ a nef linear system of positive dimension of curves of arithmetic genus 0 , and, accordingly, the Cremona classification of planar positive dimensional linear 
systems of rational curves: this is the foundation stone of our classification. Sections 7 and 8 are devoted to introducing and constructing planar linear systems that have the Cremona minimality property. As indicated in the statement of Theorem 0.2 , their main feature is that the maximal singularities of their general curve are nestled in a rather complicated way infinitely near to the point of highest multiplicity. In Section 9 we prove the announced classification results for pairs $(S, C)$ (and for plane curves $B$ ), by subdividing them into four main classes: the line, del Pezzo, ruled, and big cases, according to the behavior of the last effective adjoint system $\left|C+m K_{S}\right|$. An Appendix is devoted to quickly proving the famous Noether-Castelnuovo theorem on the generation of the Cremona group via linear and quadratic transformations, by exploiting the concept of simplicity of a curve (see [Al1], $[\mathrm{Ch}],[\mathrm{C}]$ ), which we effectively use in Sections 7 and 8 to prove our Cremona minimality results.

In Section 10 we present a few applications, the most relevant of which is the proof of Proposition 10.10, originally stated by de Franchis [DF], which classifies, up to Cremona transformations, planar linear systems of positive dimension of curves of genus 2. De Franchis's original proof is affected, as well as all papers on the subject that appeared before 1901, by a criticism raised by C. Segre [S] to Noether's original proof of Noether-Castelnuovo's theorem.

When the present research was completed, M. Mella kindly brought to our attention his preprint [MP2] in collaboration with E. Polastri, which contains, among other interesting things, similar results, though the classification there is less fine than the one we produce here (see also [MP1]).

\section{$\S 1$. Preliminaries}

\subsection{Notation and conventions}

In this paper we will work over $\mathbb{C}$.

Let $S$ be a smooth, irreducible, projective surface, simply called a surface in the sequel. We will use standard notation in surface theory; that is, $K=K_{S}$ will denote a canonical divisor, $q=q(S)$ will denote the irregularity of $S, \kappa=\kappa(S)$ will denote the Kodaira dimension, and so on. The linear equivalence of divisors will be denoted by $\equiv$.

Let $D$ be a divisor on $S$. As usual, $\mathcal{O}_{S}(D)$ will be the related invertible sheaf. We will denote by 0 the zero divisor. If $D$ is effective, it will be called a curve, and $p_{a}(D)$ will denote its arithmetic genus. 
If $A$ and $B$ are divisors on $S$, we will use the notation $A \geqslant B$ if $A-B$ is effective, or $A>B$ if $A \geqslant B$ and $A \neq B$. Recall that a divisor $D>0$ is said to be numerically connected if $D=A+B$, with $A, B>0$, implies that $A \cdot B>0$. A divisor $D$ is nef if for any curve $C$ one has $D \cdot C \geqslant 0$. We may sometimes consider $\mathbb{Q}$-divisors.

If $C$ is a smooth, irreducible curve with $p_{a}(C)=0$ and $C^{2}=-k<0$, we will say that $C$ is a $(-k)$-curve. By Castelnuovo's theorem, a $(-1)$-curve is the exceptional divisor of a blowup.

If $f: S \rightarrow S^{\prime}$ is a morphism and if $D, D^{\prime}$ are divisors on $S, S^{\prime}$, respectively, it makes sense to consider the image $f_{*}(D)$ of $D$ on $S^{\prime}$, the total transform $f^{*}\left(D^{\prime}\right)$, and the strict or proper transform of $D^{\prime}$ on $S$ (see [H, pp. 110, 425] and [Ma, page 121]).

\subsection{Infinitely near points}

Here we briefly recall some basic facts and terminology about infinitely near points, which will be commonly used in the sequel (cf., e.g., [A] and $[\mathrm{EC}])$.

Let $S$ and $S^{\prime}$ be surfaces. Any birational morphism $\sigma: S^{\prime} \rightarrow S$ is the composition of a certain number, say $n$, of blowing-ups $\sigma_{i}: S_{i} \rightarrow S_{i-1}$ at a point $p_{i} \in S_{i-1}, i=1, \ldots, n$ :

$$
\sigma: S^{\prime}=S_{n} \stackrel{\sigma_{n}}{\longrightarrow} S_{n-1} \stackrel{\sigma_{n-1}}{\longrightarrow} \cdots \stackrel{\sigma_{2}}{\longrightarrow} S_{1} \stackrel{\sigma_{1}}{\longrightarrow} S_{0}=S .
$$

Let $p \in S$ be a point. One says that $q$ is an infinitely near point to $p$ of order $n$, and we write $q>^{n} p$, if there exists a birational morphism $\sigma: S^{\prime} \rightarrow S$ as in $(1)$, such that $p_{1}=p ; \sigma_{i}\left(p_{i+1}\right)=p_{i}, i=1, \ldots, n-1$; and $q \in Z_{n}=$ $\sigma_{n}^{-1}\left(p_{n-1}\right)$. For each $i=1, \ldots, n$, let $E_{i}=\sigma_{i}^{-1}\left(p_{i}\right) \subset S_{i}$ be the exceptional curve of $\sigma_{i}$, and let $E_{i}^{\prime}$ be the strict transform of $E_{i}$ on $S^{\prime}$. If $i>j$, let $\sigma_{i, j}$ be the morphism $S_{i} \rightarrow S_{j}$. For each $i=1, \ldots, n-1$, set $Z_{i}=\sigma_{n, i+1}^{*}\left(E_{i}\right)$. According to the terminology introduced in Section $3, Z_{1}, \ldots, Z_{n-1}, Z_{n}=E_{n}$ are $(-1)$-cycles generating $\operatorname{Pic}\left(S^{\prime}\right)$ over $\operatorname{Pic}(S)$.

One says that $q$ is proximate to $p$, and we write $q \rightarrow p$, if either $q>^{1} p$ or $q>^{n} p$ with $n>1$ and $q$ lies on the strict transform $E_{1}^{\prime}$ of $E_{1}$ on $S^{\prime}$. In the latter case, one says that $q$ is satellite to $p$, and we write $q \odot p$. This may happen only if $p_{i}$ lies on the strict transform of $E_{1}$ on $S_{i-1}$, for each $i=2, \ldots, n$.

Also, $E_{1}^{\prime}, \ldots, E_{n-1}^{\prime}, E_{n}^{\prime}=E_{n}$ generate $\operatorname{Pic}\left(S^{\prime}\right)$ over $\operatorname{Pic}(S)$, and $E_{i}^{\prime}=Z_{i}-$ $\sum_{j} q_{i j} Z_{j}$, where $q_{i j}=1$ if $p_{j} \rightarrow p_{i}$ and $q_{i j}=0$ otherwise. 
In this paper, we will usually refer to points on a surface $S$, including infinitely near ones. We will say that a point $p$ is proper, and we will write $p \in S$, if $p$ is not infinitely near to any point of $S$. An infinitely near point is called free if it is not satellite (to any point).

Let now $C^{\prime}$ be a curve on $S^{\prime}$, and let $C=\sigma_{*}\left(C^{\prime}\right)$. Then $C^{\prime}=\sigma^{*}(C)-$ $\sum_{i=1}^{n} m_{i} Z_{i}$, where $m_{1}, \ldots, m_{n}$ are integers. If $C$ is a curve, that is, if $C^{\prime}$ is not contracted by $\sigma$, one says that $m_{i}, i=1, \ldots, n$, is the (virtual) multiplicity of $C$ at the point $p_{i}$. If no component of $C^{\prime}$ is contracted by $\sigma$, then, for each $i=1, \ldots, n$, one has $C^{\prime} \cdot E_{i}^{\prime} \geqslant 0$, which is equivalent to

$$
m_{i} \geqslant \sum_{j: p_{j} \rightarrow p_{i}} m_{j},
$$

which is classically known as the proximity inequality at $p_{i}$.

In any case, we may uniquely write $C^{\prime}=D^{\prime}+\sum_{i=0}^{n} h_{i} E_{i}^{\prime}$, with $D^{\prime}$ effective and $D^{\prime} \cdot E_{i}^{\prime} \geqslant 0$ (i.e., the proximity inequality at $p_{i}$ holds for $D^{\prime}$ ), for all $i=1, \ldots, n$. We say that $D^{\prime}$ is pure. One has $\sigma_{*}\left(D^{\prime}\right)=\sigma_{*}\left(C^{\prime}\right)=C$. Then $D^{\prime} \equiv \sigma^{*}(C)-\sum_{i=1}^{n} \tilde{m}_{i} Z_{i}$, where $\tilde{m}_{1}, \ldots, \tilde{m}_{n}$ are nonnegative integers, called the effective multiplicities of $C$ at $p_{1}, \ldots, p_{n}$. One may compute the $\tilde{m}_{i}$ from the $m_{i}$ by a well-known algorithm called Enriques's unloading principle (see $[\mathrm{EC}])$.

\subsection{Linear systems}

Let $S$ be a surface, and let $D$ be a divisor on it. As usual, we denote by $|D|$ the complete linear system associated to $D$, that is, $\mathbb{P}\left(H^{0}\left(S, \mathcal{O}_{S}(D)\right)\right)$.

A linear system $\mathcal{L} \subset|D|$ corresponds to a vector subspace $V \subset H^{0}(S$, $\left.\mathcal{O}_{S}(D)\right)$. Recall that $\mathcal{L}$ determines a rational map $\phi_{\mathcal{L}}: S \rightarrow \mathbb{P}^{r}=\mathbb{P}\left(V^{*}\right), r=$ $\operatorname{dim}(\mathcal{L})$. Two linear systems define the same map if they differ by divisorial fixed components. If $\mathcal{L}$ has no divisorial fixed component, then $\phi_{\mathcal{L}}$ is a morphism off the base locus of $\mathcal{L}$. A linear system $\mathcal{L}$ will be called irreducible if its general curve is irreducible.

If $\mathcal{L} \subset|D|$ and $\mathcal{L}^{\prime} \subset\left|D^{\prime}\right|$ are linear systems, corresponding to $V \subset H^{0}(S$, $\left.\mathcal{O}_{S}(D)\right)$ and $V^{\prime} \subset H^{0}\left(S, \mathcal{O}_{S}\left(D^{\prime}\right)\right)$, we will denote by $\mathcal{L} \cdot \mathcal{L}^{\prime}$ the intersection number $C \cdot C^{\prime}$, where $C \in \mathcal{L}$ and $C^{\prime} \in \mathcal{L}^{\prime}$, and by $\mathcal{L}+\mathcal{L}^{\prime}$ the linear system corresponding to the image of $V \otimes V^{\prime} \subset H^{0}\left(S, \mathcal{O}_{S}(D)\right) \otimes H^{0}\left(S, \mathcal{O}_{S}\left(D^{\prime}\right)\right)$ in $H^{0}\left(S, \mathcal{O}_{S}\left(D+D^{\prime}\right)\right)$ via the natural multiplication map. Thus we may consider the multiple linear system $i \mathcal{L}$ for all integers $i \geqslant 1$.

Let $\phi: S \rightarrow S^{\prime}$ be a dominant rational map, let $\mathcal{L}$ be a linear system on $S$, and let $C \in \mathcal{L}$ be its general curve. We will denote by $\phi_{*}(\mathcal{L})$ the 
proper image of $\mathcal{L}$, which is the linear system on $S^{\prime}$ whose general curve is the closure of the images of $\phi(x)$, where $x$ varies among the general points of all irreducible components of $C$.

One also defines the total transform $\phi_{!}(\mathcal{L})$ of $\mathcal{L}$ via $\phi$, which is defined as follows. There is a commutative diagram

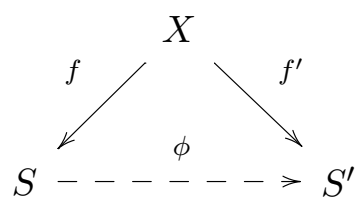

where $X$ is a surface and $f, f^{\prime}$ are birational morphisms. Then $\phi_{!}(\mathcal{L})=$ $f_{*}^{\prime}\left(f^{*}(\mathcal{L})\right)$. Note that $\phi_{*}(\mathcal{L})$ is a subsystem of $\phi_{!}(\mathcal{L})$ and that the two differ for base components which are images, via $f^{\prime}$, of exceptional divisors of $f$.

Consider a sequence of blowing-ups as in (1), and let $\mathcal{L}^{\prime}$ be a linear system on $S^{\prime}$. One can define, as in Section 1.2, virtual and effective multiplicities of $\mathcal{L}$ at the points $p_{1}, \ldots, p_{n}$ which one blows up.

Given $p_{1}, \ldots, p_{n}$ proper or infinitely near points of $\mathbb{P}^{2}$, and given positive integers $m_{1}, \ldots, m_{r}$ for each one of them, we denote by

$$
\mathcal{L}\left(d ; m_{1}, m_{2}, \ldots, m_{r}\right)
$$

the linear system $\mathcal{L} \subseteq\left|\mathcal{O}_{\mathbb{P}^{2}}(d)\right|$ of curves of degree $\operatorname{deg}(\mathcal{L})=d$ with assigned base points $p_{1}, \ldots, p_{n}$ and with assigned, or virtual, multiplicities $m_{1}, \ldots$, $m_{n}$. It may happen that the system $\mathcal{L}$ has further base points, fixed components, and higher multiplicities than the assigned ones. The last phenomenon means exceptional divisors as fixed components, if one looks at the system on the blowup of $\mathbb{P}^{2}$ at the assigned base points.

We will usually assume that $m_{1} \geqslant m_{2} \geqslant \cdots \geqslant m_{r}$. We will use the exponential notation $m_{i}^{e_{i}}$ in case of $e_{i}$ points of multiplicity $m_{i}$.

In cases where we want to specify that $e_{i}$ points of multiplicity $m_{i}, i=$ $1, \ldots, l$, are infinitely near (resp., proximate) to a point of multiplicity $m$, we will write

$$
\begin{gathered}
\mathcal{L}\left(\ldots,\left(m,\left\{m_{1}^{e_{1}}, m_{2}^{e_{2}}, \ldots, m_{l}^{e_{l}}\right\}\right), \ldots\right) \\
{\left[\text { resp., } \mathcal{L}\left(\ldots,\left(m,\left[m_{1}^{e_{1}}, m_{2}^{e_{2}}, \ldots, m_{l}^{e_{l}}\right]\right), \ldots\right)\right] .}
\end{gathered}
$$

Recall that the virtual dimension of the linear system $\mathcal{L}=\mathcal{L}\left(d ; m_{1}, \ldots\right.$, $\left.m_{r}\right)$ is $v(\mathcal{L})=d(d+3) / 2-\sum_{i=1}^{r} m_{i}\left(m_{i}+1\right) / 2$. One has $\operatorname{dim}(\mathcal{L}) \geqslant$ $\max \{v(\mathcal{L}),-1\}$, and if the equality holds, the system is called nonspecial. 


\subsection{Cremona transformations}

A linear system $\mathcal{L}$ of plane curves, with no divisorial fixed components, is called a net if $\operatorname{dim}(\mathcal{L})=2$. If, in addition, the map $\phi_{\mathcal{L}}: \mathbb{P}^{2}-\rightarrow \mathbb{P}^{2}$ is a Cremona transformation, that is, it is birational, the net is called homaloidal. In that case, the general curve $C$ of $\mathcal{L}$ is irreducible and rational. If $d$ is the degree of $\mathcal{L}$, one says that $\phi_{\mathcal{L}}$ has degree $d$. Cremona transformations of degree 1 are projective or linear transformations.

An irreducible net $\Lambda$ of type $\mathcal{L}\left(\delta ; \delta-1,1^{2 \delta-2}\right), \delta \geqslant 2$, is homaloidal, and the corresponding birational map $\phi_{\Lambda}: \mathbb{P}^{2} \rightarrow \mathbb{P}^{2}$ is called a de Jonquières transformation of degree $\delta$ centered at the base points of $\Lambda$. If $\delta=2$, the map $\phi_{\Lambda}$ is a quadratic transformation.

Any homaloidal net $\mathcal{L}\left(\delta ; \alpha_{0}, \alpha_{1}, \ldots, \alpha_{r}\right)$ is such that

$$
\delta^{2}-1=\sum_{i=0}^{r} \alpha_{i}^{2}, \quad 3(\delta-1)=\sum_{i=0}^{r} \alpha_{i}
$$

Recall the following famous theorem.

Theorem 1.1 (Noether-Castenuovo). Every Cremona transformation of the plane is the composition of finitely many linear and quadratic transformations.

In Appendix A we give a proof of this theorem by induction on the simplicity of homaloidal nets, which we now introduce and will use later.

Let $\mathcal{L}=\mathcal{L}\left(d ; m_{0}, m_{1}, \ldots, m_{r}\right)$ be a planar linear system with $d \geqslant m_{0} \geqslant$ $m_{1} \geqslant \cdots \geqslant m_{r} \geqslant 1$, and let $p_{i}$ be the point of multiplicity $m_{i}$ of $\mathcal{L}$. (If there is no $m_{i}$, set $r=-1$.) We set $m_{r+1}=0$ and $m_{-1}=\infty$. The simplicity of $\mathcal{L}$ is the triplet $\left(k_{\mathcal{L}}, h_{\mathcal{L}}, s_{\mathcal{L}}\right)$ of integers defined as follows:

$$
\begin{gathered}
k_{\mathcal{L}}=d-m_{0}, \quad m_{h_{\mathcal{L}}}>\frac{k_{\mathcal{L}}}{2} \geqslant m_{h_{\mathcal{L}}+1}, \\
s_{\mathcal{L}}=\sharp\left\{p_{i} \mid 1 \leqslant i \leqslant h \text { and } p_{i} \odot p_{0}\right\} .
\end{gathered}
$$

One says that $\mathcal{L}^{\prime}$ is simpler than $\mathcal{L}$ if the simplicity of $\mathcal{L}^{\prime}$ is lexicographically smaller than the one of $\mathcal{L}$. The simplicity of a Cremona transformation $\phi: \mathbb{P}^{2} \rightarrow \mathbb{P}^{2}$ is the one of the homaloidal net defining $\phi$. A de Jonquières transformation of degree $\delta$ has simplicity $(1,2 \delta-2, s)$, with $s \leqslant \delta-1$. 


\section{§2. Birational equivalence of pairs and Cremona minimality}

Let $(S, \mathcal{L})$ be a pair with $S$ a surface and $\mathcal{L}$ a linear system on it. If $(S, \mathcal{L})$, $\left(S^{\prime}, \mathcal{L}^{\prime}\right)$ are two such pairs, we say that they are birationally equivalent, and we write $(S, \mathcal{L}) \sim\left(S^{\prime}, \mathcal{L}^{\prime}\right)$, if there is a birational map $\phi: S \rightarrow S^{\prime}$ such that $\mathcal{L}^{\prime}=\phi_{*}(\mathcal{L}), \mathcal{L}=\phi_{*}^{-1}\left(\mathcal{L}^{\prime}\right)$, and $\phi$ is not constant on each irreducible component of the divisorial part of the base locus of $\mathcal{L}$. If, moreover, $\phi: S \rightarrow S^{\prime}$ is an isomorphism, the pairs $(S, \mathcal{L}),\left(S^{\prime}, \mathcal{L}^{\prime}\right)$ are called isomorphic. Birational equivalence is an equivalence relation. Any pair in a class is called a model of the class.

If $(S, \mathcal{L}) \sim\left(S^{\prime}, \mathcal{L}^{\prime}\right)$, then $\operatorname{dim}(\mathcal{L})=\operatorname{dim}\left(\mathcal{L}^{\prime}\right)$. If $\operatorname{dim}(\mathcal{L})=0$, we have the notion of birational equivalence of pairs $(S, C)$, where $C$ is a curve on $S$.

If $(S, \mathcal{L}) \sim\left(S^{\prime}, \mathcal{L}^{\prime}\right)$, then the images of $\phi_{\mathcal{L}}$ and $\phi_{\mathcal{L}^{\prime}}$ are projectively equivalent. The converse is also true, provided $\phi_{\mathcal{L}}$ and $\phi_{\mathcal{L}^{\prime}}$ are birational to their images.

Given a pair $(S, \mathcal{L})$, with $S$ rational, one can consider all models of $(S, \mathcal{L})$ of the form $\left(\mathbb{P}^{2}, \mathcal{L}^{\prime}\right)$. Those with minimal $\operatorname{deg}\left(\mathcal{L}^{\prime}\right)$ will be called Cremona minimal, and those with $\operatorname{deg}\left(\mathcal{L}^{\prime}\right)$ will be called the Cremona degree $\operatorname{cdeg}(\mathcal{L})$ of $\mathcal{L}$. This definition includes the one of Cremona degree and Cremona minimality for curves. It is clear that $\operatorname{cdeg}(\mathcal{L}) \geqslant \operatorname{cdeg}(C)$, where $C$ is the general member of $\mathcal{L}$. As we shall see, it may happen that strict inequality holds.

If $(S, \mathcal{L})$ is birationally equivalent to $\left(\mathbb{P}^{2}, \mathcal{L}\left(d ; m_{1}^{e_{1}}, \ldots, m_{r}^{e_{r}}\right)\right)$, we say that $\mathcal{L}$ is of Cremona type $\left(d ; m_{1}^{e_{1}}, \ldots, m_{r}^{e_{r}}\right)$. If $d=\operatorname{cdeg}(\mathcal{L})$, we say that $\mathcal{L}$ is of minimal Cremona type $\left(d ; m_{1}^{e_{1}}, \ldots, m_{r}^{e_{r}}\right)$.

Consider a linear system $\mathcal{L}=\mathcal{L}\left(d ; m_{1}, \ldots, m_{r}\right)$ and a Cremona transformation $\phi_{\Lambda}: \mathbb{P}^{2} \rightarrow \mathbb{P}^{2}$ determined by the homaloidal net $\Lambda=\mathcal{L}\left(\delta ; \alpha_{1}, \ldots\right.$, $\left.\alpha_{s}\right)$. At the cost of taking some $\alpha_{i}=0$, we may assume that $s \geqslant r$. Moreover, we assume that the assigned base points of $\mathcal{L}$ coincide with the points of multiplicities $\alpha_{1}, \ldots, \alpha_{r}$ of $\Lambda$. One has

$$
\operatorname{deg}\left(\phi_{*}(\mathcal{L})\right) \leqslant d \delta-\sum_{i=1}^{r} \alpha_{i} m_{i},
$$

and the equality holds if $\mathcal{L}$ has no base points off the assigned ones and if its effective multiplicities equal the assigned ones. We denote by $\operatorname{vdeg}_{\phi}(\mathcal{L})$ the right-hand side of $(5)$ and call it the virtual degree of $\phi_{*}(\mathcal{L})$.

The following lemma gives useful criteria for Cremona minimality. 
Lemma 2.1. Planar linear systems $\mathcal{L}$ of the following types are Cremona minimal:

(i) $\mathcal{L}(d ; n, m)$, with $d \geqslant n+m$ and $n \geqslant m \geqslant 0$;

(ii) $\mathcal{L}\left(d ;\left(m,\left[m_{1}, \ldots, m_{h}\right]\right)\right)$, where $h \geqslant 1, m_{1} \geqslant m_{2} \geqslant \cdots \geqslant m_{h} \geqslant 1, m \geqslant$ $m_{1}+\cdots+m_{h}, d \geqslant m+m_{1}$, and the point $p_{i}, i=1, \ldots, h$, of multiplicity $m_{i}$ is infinitely near of order 1 to the point $p$ of multiplicity $m$.

Moreover, a Cremona transformation $\phi: \mathbb{P}^{2} \rightarrow-\rightarrow \mathbb{P}^{2}$ such that $\mathcal{L}^{\prime}=\phi_{*}(\mathcal{L})$ has degree $d$ is linear except:

- in case (i), $d=n+m$, in which $\phi$ may be a de Jonquières transformation of degree $\delta \geqslant 2$ centered at the base points of $\mathcal{L}$, and $\delta=2$ unless $m=0$, that is, unless $\mathcal{L}$ is composed of a pencil of lines;

- in case (ii), in which $\phi$ may be a de Jonquières transformation of degree $\delta \leqslant h^{\prime}+1$, where $h^{\prime}$ is the maximum such that $d-m=m_{1}=\cdots=m_{h^{\prime}}$, and $\phi$ is centered at $p$ and at $\delta-1$ points among $p_{1}, \ldots, p_{h^{\prime}}$.

In all cases $\mathcal{L}^{\prime}$ is of the same Cremona type as $\mathcal{L}$.

Proof. (i) One sees that $\mathcal{L}$ has no unassigned base point. Suppose that the homaloidal net corresponding to $\phi$ has degree $\delta$ and multiplicities $\alpha, \beta$ at the points of multiplicities $n, m$ of $\mathcal{L}$. We may assume that $\delta>1, \alpha \geqslant \beta$ and $\delta \geqslant \alpha+\beta$, and $\delta-1 \geqslant \alpha$. Then $\phi$ maps $\mathcal{L}$ to a linear system $\mathcal{L}^{\prime}$ of degree

$$
\delta d-\alpha n-\beta m \geqslant \delta d-\alpha(n+m) \geqslant(\delta-\alpha) d \geqslant d
$$

If the equality holds, then either $\beta=m=0, n=d$, and $\alpha=\delta-1$, or $\alpha=$ $\beta=\delta-1=1$ and $d=n+m$, proving the assertion in case (i).

(ii) By blowing up $p$, working on $\mathbb{F}_{1}$, and applying Bertini's theorem, one sees that $\mathcal{L}$ has no unassigned base point and that its general curve is irreducible, with multiplicities at the base points equal to the assigned ones. Suppose that the homaloidal net corresponding to $\phi$ has degree $\delta>1$ and multiplicities $\alpha$ and $\alpha_{i}$, respectively, at $p$ and at $p_{i}, i=1, \ldots, h$. Then $\delta-1 \geqslant \alpha \geqslant \sum_{i=1}^{h} \alpha_{i}$, by (2), and $\alpha_{i} \geqslant 0$ for each $i$. Thus $\phi$ maps $\mathcal{L}$ to a linear system $\mathcal{L}^{\prime}$ of degree

$$
\begin{aligned}
\delta d-\alpha m-\sum_{i=1}^{h} \alpha_{i} m_{i} & \geqslant \delta d-\alpha m-(d-m) \sum_{i=1}^{h} \alpha_{i} \\
& \geqslant \delta d-\alpha m-(d-m) \alpha=(\delta-\alpha) d \geqslant d .
\end{aligned}
$$


If equality holds, then $\alpha=\delta-1, \alpha_{i} \leqslant 1$ for each $i, d=m+m_{i}$ whenever $\alpha_{i}=1$, and there are exactly $\delta-1$ indexes $i$ such that $\alpha_{i}=1$. This proves the assertion in case (ii).

If $\mathcal{L}$ is a linear system of plane curves with no multiple fixed components, then there is a birational morphism $f: S \rightarrow \mathbb{P}^{2}$ such that the proper transform of the general curve $C$ of $\mathcal{L}$ is smooth. Let $\mathcal{L}^{\prime}$ be the linear system on $S$ such that $\left(S, \mathcal{L}^{\prime}\right) \sim\left(\mathbb{P}^{2}, \mathcal{L}\right)$ via $f^{-1}$. We say that $\mathcal{L}$ is complete if $\mathcal{L}^{\prime}$ is complete. We will denote by $\operatorname{ad}_{m}(\mathcal{L})$ the linear system $f_{*}\left(\left|C+m K_{S}\right|\right)$, and we call it the $m$-adjoint linear system of $\mathcal{L}$. This system is independent on $f$. If $\mathcal{L}=\{C\}$ has dimension zero, we write $\operatorname{ad}_{m}(C)$. In case $m=1$, we write $\operatorname{ad}(\mathcal{L})$ and call it the adjoint linear system of $\mathcal{L}$. Note that $\operatorname{deg}\left(\operatorname{ad}_{m}(\mathcal{L})\right)=\operatorname{deg}(\mathcal{L})-3 m$. One similarly defines $m$-adjoint linear systems of $\mathcal{L}$ for a linear system $\mathcal{L}$ with no multiple fixed components, on any surface.

REMARK 2.2. Whenever $\operatorname{ad}_{m}(\mathcal{L})$ and $\mathcal{L}$ are not empty and $\operatorname{ad}_{m}(\mathcal{L})$ is without fixed component and Cremona minimal, then also $\mathcal{L}$ is Cremona minimal (cf. Proposition 4.4).

Using adjoints, one may easily prove a very useful, classical result due to Jung [J] (cf. [Coo, pages 402-403]).

Theorem 2.3 (Jung $[\mathrm{J}])$. A linear system $\mathcal{L}=\mathcal{L}\left(d ; m_{1}, m_{2}, m_{3}, \ldots\right)$, without multiple fixed components, with $m_{1} \geqslant m_{2} \geqslant m_{3} \geqslant \cdots$ and $d \geqslant m_{1}+m_{2}+$ $m_{3}$, is Cremona minimal.

Proof. By Lemma 2.1, we may assume that $m_{3}>0$. Then $\operatorname{ad}_{m_{3}}(\mathcal{L})=$ $\mathcal{L}\left(d-3 m_{3} ; m_{1}-m_{3}, m_{2}-m_{3}\right)$ is Cremona minimal, again by Lemma 2.1, and so is $\mathcal{L}$ (see Remark 2.2).

Furthermore, Lemma 2.1 implies the following.

Corollary 2.4. Let $\mathcal{L}$ be as in Theorem 2.3. If $d>m_{1}+m_{2}+m_{3}$ and if $\phi: \mathbb{P}^{2} \rightarrow \mathbb{P}^{2}$ is a Cremona transformation such that $\phi_{*}(\mathcal{L})$ has degree $d$, then $\phi$ is linear.

A linear system $\mathcal{L}$ satisfying the hypotheses of Theorem 2.3 will be called of Noether type.

\section{$\S 3$. Properties of $(-1)$-cycles}

Let $S$ be a surface, and let $Z>0$ be a divisor on $S$. We will say that $Z$ is a $(-1)$-cycle if there is a surface $S^{\prime}$ and a birational morphism $f: S \rightarrow S^{\prime}$ 
such that $f(Z)$ is a point $p \in S^{\prime} ; Z$ is the scheme theoretical fiber of $f$ over $p$, that is, $\mathcal{O}_{S}(-Z) \simeq f^{*}\left(\mathcal{I}_{p \mid S^{\prime}}\right)$; and $f: S^{\prime}-Z \rightarrow S-\{p\}$ is an isomorphism. In this case we will say that $f$ blows down $Z$.

The structure of a $(-1)$-cycle has been classically studied by Barber and Zariski [BZ] and Franchetta [F1]. We will not need it here.

If $Z$ is irreducible, then it is the exceptional divisor of a blowup. Otherwise, $f$ is the composition of blowups

$$
f: S=S_{0} \rightarrow S_{1} \rightarrow \cdots \rightarrow S_{n-1} \rightarrow S_{n}=S^{\prime} .
$$

If $j>i$, denote by $f_{i, j}$ the morphism $S_{i} \rightarrow S_{j}$, and denote by $Z_{i, j}$ the corresponding (-1)-cycle on $S_{i}$. Then $Z$ is the total transform of $Z_{i, n}$ via the map $f_{0, i}$, for all $i=1, \ldots, n-1$. We will say that each $Z_{0, j}$ is $f$-exceptional.

The proof of the following lemma is trivial.

LEMMA 3.1. In the above setting, if $0 \leqslant i<h<j \leqslant n$, one has $\left(f_{i, h}\right)_{*}\left(Z_{i, j}\right)=Z_{h, j}$ and $Z_{i, j} \cdot Z_{i, h}=0$.

The following lemmas are classical (see [BZ], [F1]).

Lemma 3.2. Let $Z$ be a (-1)-cycle on a surface $S$, and let $f: S \rightarrow S^{\prime}$ be the birational morphism blowing down $Z$. Then

$$
K_{S} \equiv f^{*}\left(K_{S^{\prime}}\right)+\sum_{i=1}^{n} Z_{0, i}
$$

Proof. Proceed by induction on the number $n$ of blowups appearing in the sequence $(6)$.

LEMMA 3.3. If $Z$ is a (-1)-cycle on a surface $S$, then

$$
K_{S} \cdot Z=Z^{2}=-1 ; \quad \text { thus } p_{a}(Z)=0 \text {. }
$$

Proof. Since $Z$ is the total transform of the exceptional curve $Z_{n-1, n}$, one has $Z^{2}=-1$. By intersecting both sides of (7) with $Z$ and taking into account Lemma 3.1, one finds $K_{S} \cdot Z=-1$.

By Zariski's main theorem, a (-1)-cycle is topologically connected. More precisely (see $[\mathrm{F} 2],[\mathrm{F} 3]$ ), we have the following.

LEMMA 3.4. A (-1)-cycle is numerically connected. 
Proof. Let $Z$ be a $(-1)$-cycle, and let $Z=A+B$ with $A, B>0$. Suppose that $A \cdot B<0$. By Grauert's criterion (see [B, Corollary 2.7]), the intersection matrix of $Z$ is negative definite. Thus we have $-1=Z^{2}=$ $A^{2}+B^{2}+2 A \cdot B \leqslant-4$, a contradiction.

Franchetta [F2] proved that any numerically connected curve $Z$ on a surface $S$ with Kodaira dimension $\kappa(S) \geqslant 0$ verifying (8) is a (-1)-cycle. As noted by Nagata [Na2, page 282], the assertion is no longer true if $\kappa(S)=$ $-\infty$. Nagata, however, misunderstands Franchetta's statement.

In what follows we will need the following technical lemmas.

Lemma 3.5. Let $Z, Z^{\prime}$ be distinct (-1)-cycles on the surface $S$, and assume that the intersection matrix of the components of $Z+Z^{\prime}$ is negative definite. Then

(i) $Z \cdot Z^{\prime} \geqslant 0$;

(ii) if $Z \cdot Z^{\prime}=0$ and if $f: S \rightarrow S^{\prime}$ is the birational morphism blowing down $Z$, then either $f_{*}\left(Z^{\prime}\right)=0$, which happens if and only if $Z>Z^{\prime}$, or $f_{*}\left(Z^{\prime}\right)$ is a $(-1)$-cycle on $S^{\prime}$.

Proof. Assertion (i) is clear if $Z, Z^{\prime}$ have no common component. Let us proceed by induction on the number of common components of $Z$ and $Z^{\prime}$.

Consider the map $f: S \rightarrow S^{\prime}$ blowing down $Z$, and suppose that it is a sequence as in (6). By Lemma 3.1 , we have $Z \cdot Z_{0,1}=0$.

Assume first that $Z_{0,1} \leqslant Z^{\prime}$. If $Z_{0,1}=Z^{\prime}$, then $Z \cdot Z^{\prime}=0$. Otherwise, $Z_{1}^{\prime}:=\left(f_{0,1}\right)_{*}\left(Z^{\prime}\right)$ is a $(-1)$-cycle $Z_{1}^{\prime}$ on $S_{1}$ and $Z^{\prime}=f_{0,1}^{*}\left(Z_{1}^{\prime}\right)$. By induction, we have

$$
Z \cdot Z^{\prime}=f_{0,1}^{*}\left(Z_{1, n}\right) \cdot f_{0,1}^{*}\left(Z_{1}^{\prime}\right)=Z_{1, n} \cdot Z_{1}^{\prime} \geqslant 0 .
$$

Assume now that $Z_{0,1}$ is not contained in $Z^{\prime}$. One has $Z_{0,1} \cdot Z^{\prime}=0$; otherwise, $\left(Z^{\prime}+Z_{0,1}\right)^{2} \geqslant 0$, against the negativity assumption on the intersection matrix of the components of $Z+Z^{\prime}$. Then $Z_{0,1}$ and $Z^{\prime}$ have no points in common; therefore, $\left(f_{0,1}\right)_{*}\left(Z^{\prime}\right)$ is a $(-1)$-cycle on $S_{1}$, and we can repeat the argument. After a finite number of steps, we accomplish the proof of (i). The proof of (ii) is analogous and may be left to the reader.

Lemma 3.6. Let $Z, Z^{\prime}$ be distinct (-1)-cycles on the surface $S$. If $Z \cdot Z^{\prime}>$ 0 , then

(i) if $q(S)=0$, there is some divisor $D \leqslant Z+Z^{\prime}$ which moves in a linear system of positive dimension on $S$; 
(ii) if $q(S)>0$, then the Albanese map of $S$ maps $S$ to a curve, and $Z+Z^{\prime}$ is a rational multiple of a fiber.

Proof. If $q=0$, by the Riemann-Roch theorem we have $h^{0}\left(S, \mathcal{O}_{S}(Z+\right.$ $\left.\left.Z^{\prime}\right)\right) \geqslant 1+Z \cdot Z^{\prime} \geqslant 2$, and the assertion follows.

If $q>0$, then the Albanese map contracts $Z+Z^{\prime}$ to a point. Hence, the intersection matrix of the components of $Z+Z^{\prime}$ is negative semidefinite (see [B, Corollaries 2.6 and 2.7]). Since $\left(Z+Z^{\prime}\right)^{2}=2\left(Z \cdot Z^{\prime}-1\right.$ ), we have $Z \cdot Z^{\prime}=1$ and $\left(Z+Z^{\prime}\right)^{2}=0$, and the assertion follows from Zariski's lemma (see [B, Corollary 2.6]).

\section{§4. Basic properties of adjoint linear systems}

\subsection{Nefness property of $m$-adjoint linear systems}

The following results are essentially known to the experts. Since we have not found a proper reference, we quickly present them here.

Proposition 4.1. Let $C$ be a divisor on a surface $S$ such that either

(i) $C$ is nef; or

(ii) $C$ is effective, irreducible, and not a $(-k)$-curve, with $1 \leqslant k \leqslant 3$.

Let $m>0$ be an integer. Suppose that $\left|C+m K_{S}\right| \neq \emptyset$ and that $m \geqslant 2$ if we are in case (ii) and $C$ is rational.

Then there is a surface $S^{\prime}$ and a birational morphism $f: S \rightarrow S^{\prime}$ such that $\left|C^{\prime}+m K_{S^{\prime}}\right|$ is nef, where $C^{\prime}=f_{*}(C)$, and

$$
h^{0}\left(S, \mathcal{O}_{S}\left(C+m K_{S}\right)\right)=h^{0}\left(S^{\prime}, \mathcal{O}_{S^{\prime}}\left(C^{\prime}+m K_{S^{\prime}}\right)\right) .
$$

Proof. If $C+m K_{S}$ is nef, the assertion is clear. Suppose that there are irreducible curves $\Theta>0$ such that $\Theta \cdot\left(C+m K_{S}\right)<0$. Since $C+m K_{S} \geqslant 0$, there are only finitely many such curves, and each of them has $\Theta^{2}<0$. We claim that they are $(-1)$-curves. Indeed, if (i) holds, then $0>\Theta \cdot(C+$ $\left.m K_{S}\right)=\Theta \cdot C+m\left(\Theta \cdot K_{S}\right) \geqslant m\left(\Theta \cdot K_{S}\right)$; hence, $\Theta \cdot K_{S}<0$, and therefore $\Theta^{2}=-1$ and $p_{a}(\Theta)=0$. If (ii) holds, one has $C \cdot \Theta \geqslant 0$, and therefore the same conclusion holds. Indeed, if $C \cdot \Theta<0$, then $C=\Theta$ and $0>\Theta \cdot(C+$ $\left.m K_{S}\right)=2 m\left(p_{a}(C)-1\right)-C^{2}(m-1)$. This implies that $p_{a}(C)=0$; hence, $m \geqslant 2$ and $C^{2}>-2-2 /(m-1)$, and thus $C^{2} \geqslant-3$, a contradiction.

Let $\Theta$ be a $(-1)$-curve such that $\Theta \cdot\left(C+m K_{S}\right)<0$. Set $\Theta \cdot C=i \geqslant 0$, and note that $i<m K_{S} \cdot \Theta=m$. We have a birational morphism $\pi: S \rightarrow S_{1}$ blowing down $\Theta$ to a smooth point $p$. Set $C_{1}=\pi_{*}(C)$. Then $C=\pi^{*}\left(C_{1}\right)-$ 
$i \Theta$, and $K_{S} \equiv \pi^{*}\left(K_{S_{1}}\right)+\Theta ;$ hence,

$$
C+m K_{S} \equiv \pi^{*}\left(C_{1}+m K_{S_{1}}\right)+(m-i) \Theta,
$$

and

$$
h^{0}\left(S_{1}, \mathcal{O}_{S_{1}}\left(C_{1}+m K_{S_{1}}\right)\right)=h^{0}\left(S, \mathcal{O}_{S}\left(C+m K_{S}\right)\right) .
$$

So $C_{1}+m K_{S_{1}}$ is effective. By repeating this argument, we see that there is a sequence of blowdowns as in (6), such that $C^{\prime}+m K_{S^{\prime}}$ is nef, where $C^{\prime}=f_{*}(C)$ and $(9)$ holds.

Consider all $f$ exceptional $(-1)$-cycles. We may index them with two indices as $\Theta_{i, j}$, where $i=C \cdot \Theta_{i, j}$ and $j=1, \ldots, h_{i}$. Then

$$
C=f^{*}\left(C^{\prime}\right)-\sum_{i} \sum_{j=1}^{h_{i}} i \Theta_{i, j} \quad \text { and } \quad K_{S}=K_{S^{\prime}}+\sum_{i} \sum_{j=1}^{h_{i}} \Theta_{i, j} .
$$

The following proposition specifies the Zariski decomposition of the adjoint systems we are interested in.

Proposition 4.2. Assume the same hypotheses of Proposition 4.1. Then we have a unique expression

$$
C+m K_{S} \equiv P+\sum_{i=0}^{m-1} \sum_{j=1}^{h_{i}}(m-i) \Theta_{i, j}
$$

where $P$ is nef and the $\Theta_{i, j}$ are $(-1)$-cycles such that

(i) $P \cdot \Theta_{i, j}=0$, for each $(i, j)$;

(ii) $\Theta_{i, j} \cdot \Theta_{h, k}=0$, for $(h, k) \neq(i, j)$;

(iii) the intersection matrix of the irreducible components of

$$
N=\sum_{i=0}^{m-1} \sum_{j=1}^{h_{i}}(m-i) \Theta_{i, j}
$$

is negative definite;

(iv) $C \cdot \Theta_{i, j}=i$, for each $(i, j)$; and

(v) $h^{0}\left(S, \mathcal{O}_{S}\left(C+m K_{S}\right)\right)=h^{0}\left(S, \mathcal{O}_{S}(P)\right)$. 
Proof. It follows from Proposition 4.1 and from (10). Note that $P=$ $f^{*}\left(C^{\prime}+m K_{S^{\prime}}\right)$ is nef. Then (i) is clear, (ii) follows from Lemma 3.1, (iii) follows from [B, Corollary 2.7], (iv) holds by definition, and (v) follows from Proposition 4.1. Since $i-m=\Theta_{i, j} \cdot\left(C+m K_{S}\right)<0$, we have $i<m$.

As announced, (11) is nothing else than the Zariski decomposition of $C+m K_{S}$ (see [B, Chapter 14]). As such, it is unique.

The divisors $N, P$ appearing in Proposition 4.2(iii) are the negative part and the nef part, respectively, of $C+m K_{S}$.

REMARK 4.3. The decomposition (11) is stable under birational morphisms, in the following sense. Let $X$ be a surface, let $f: X \rightarrow S$ be a birational morphism, and let $\Gamma$ be the proper transform on $X$ of the curve $C$ on $S$.

Let $\Theta_{i, j}$ be the $f$-exceptional $(-1)$-cycles on $X$, indexed in such a way that $\Theta_{i, j} \cdot \Gamma=i, j=1, \ldots, k_{i}$. Then

$$
\Gamma=f^{*}(C)-\sum_{i} \sum_{j=1}^{k_{i}} i \Theta_{i, j} \quad \text { and } \quad K_{X} \equiv f^{*}\left(K_{S}\right)+\sum_{i} \sum_{j=1}^{k_{i}} \Theta_{i, j}
$$

so that

$$
\Gamma+m K_{X} \equiv f^{*}\left(C+m K_{S}\right)+\sum_{i} \sum_{j=1}^{k_{i}}(m-i) \Theta_{i, j} .
$$

From this we deduce that

$$
h^{0}\left(S, \mathcal{O}_{S}\left(C+m K_{S}\right)\right) \geqslant h^{0}\left(X, \mathcal{O}_{X}\left(\Gamma+m K_{X}\right)\right),
$$

and equality holds if each $i$ is at most $m$. Assume that this is true. This is the case if $C$ has points of multiplicity at most $m$. Then, substituting for $C+m K_{S}$ the expression given by (11), we find the analogous decomposition for $\Gamma+m K_{X}$.

More generally, if $n \geqslant 1$ is an integer, we have

$$
n \Gamma+m K_{X} \equiv f^{*}\left(n C+m K_{S}\right)+\sum_{i} \sum_{j=1}^{k_{i}}(m-n i) \Theta_{i, j}
$$

and

$$
h^{0}\left(S, \mathcal{O}_{S}\left(n C+m K_{S}\right)\right) \geqslant h^{0}\left(X, \mathcal{O}_{X}\left(n \Gamma+m K_{X}\right)\right),
$$

with equality if $m \geqslant n i$ for all $i$. 


\subsection{Adjoint systems and birational transformations}

Suppose that $(S, C) \sim\left(S^{\prime}, C^{\prime}\right)$ via the birational map $\phi: S \rightarrow S^{\prime}$. Then there is a commutative diagram

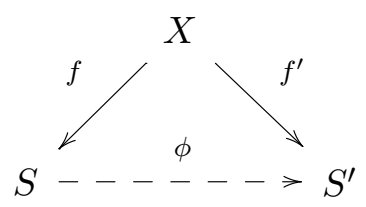

where $X$ is a surface, $f, f^{\prime}$ are birational morphisms, and there is a curve $\Gamma$ on $X$ such that $f_{*}(\Gamma)=C, f_{*}^{\prime}(\Gamma)=C^{\prime}$.

As we saw,

$$
\begin{aligned}
\Gamma+m K_{X} & \equiv f^{*}\left(C+m K_{S}\right)+\sum_{i} \sum_{j=1}^{h_{i}}(m-i) \Theta_{i, j} \\
& \equiv f^{\prime *}\left(C^{\prime}+m K_{S^{\prime}}\right)+\sum_{u} \sum_{v=1}^{h_{u}^{\prime}}(m-u) \Theta_{u, v}^{\prime}
\end{aligned}
$$

and therefore

$$
C^{\prime}+m K_{S^{\prime}} \equiv \phi_{!}\left(C+m K_{S}\right)+\sum_{i} \sum_{j=1}^{h_{i}}(m-i) f_{*}^{\prime}\left(\Theta_{i, j}\right) .
$$

Proposition 4.4. In the above setting, assume that $C, C^{\prime}$ verify the hypotheses of Proposition 4.1 and have points of multiplicity at most $m$. Then

$$
h^{0}\left(S, \mathcal{O}_{S}\left(C+m K_{S}\right)\right)=h^{0}\left(S^{\prime}, \mathcal{O}_{S^{\prime}}\left(C^{\prime}+m K_{S^{\prime}}\right)\right)
$$

If, in addition, $C$ and $C^{\prime}$ have points of multiplicity at most $m-1$, then

$$
C^{\prime}+m K_{S^{\prime}} \equiv \phi_{!}\left(C+m K_{S}\right)+\sum_{i=1}^{m-1} \sum_{\ell=1}^{k_{i}}(m-i) \theta_{i, \ell}
$$

where $\theta_{i, j}$ are $(-1)$-cycles on $S^{\prime}$ such that $C^{\prime} \cdot \theta_{i, \ell}=i$, which are the images of $f$-exceptional $(-1)$-cycles.

Proof. The first assertion follows from Remark 4.3.

As for the second assertion, from (12) we see that all the $f^{\prime}$-exceptional $(-1)$-cycles $\Theta_{u, v}^{\prime}$ sit in the negative part of $\Gamma+m K_{X}$. Then $\Theta_{i, j} \cdot \Theta_{u, v}^{\prime}=0$. By Lemma 3.5(ii), we see that $\theta_{i, j}:=f_{*}^{\prime}\left(\Theta_{i, j}\right)$ are either zero or $(-1)$-cycles. Moreover, $\theta_{i, j} \cdot \phi_{!}\left(C+m K_{S}\right)=0$, which proves that $C^{\prime} \cdot \theta_{i, \ell}=i$. 
In the hypotheses of Proposition 4.4, we set

$$
a_{m}(C):=h^{0}\left(S, \mathcal{O}_{S}\left(C+m K_{S}\right)\right),
$$

which is a birational invariant of the pair $(S, C)$.

REMARK 4.5. Let $n \geqslant 1$ be an integer. Assume that $C, C^{\prime}$ verify the hypotheses of Proposition 4.1, from which we keep the notation, and have points of multiplicity at most $[m / n]$. In particular, $m \geqslant n$. Assume that $h^{0}\left(S, \mathcal{O}_{S}\left(n C+m K_{S}\right)\right)>0$. Then

$$
n C^{\prime}+m K_{S^{\prime}} \equiv \phi_{!}\left(n C+m K_{S}\right)+\sum_{i=1}^{[m / n]} \sum_{\ell=1}^{k_{i}}(m-n i) \theta_{i, \ell}
$$

and

$$
h^{0}\left(S, \mathcal{O}_{S}\left(n C+m K_{S}\right)\right)=h^{0}\left(S^{\prime}, \mathcal{O}_{S^{\prime}}\left(n C^{\prime}+m K_{S^{\prime}}\right)\right)
$$

so that

$$
a_{m}(n C):=h^{0}\left(S, \mathcal{O}_{S}\left(n C+m K_{S}\right)\right)
$$

is also a birational invariant of the pair $(S, C)$ (see [I2]).

More specifically, the projective isomorphism class of the image of $S$ via $\phi_{\left|n C+m K_{S}\right|}$ is a birational invariant.

REMARK 4.6. All of the above results hold for pairs $(S, \mathcal{L})$ with $\mathcal{L}$ a linear system as well.

Let $(S, C)$ be a pair as usual, with $S$ smooth and $C$ with points of multiplicity at most $m$. The expert reader in higher dimensional birational geometry will commonly express this by saying that the pair $(S, C / m)$ has canonical singularities, and actually terminal singularities if $C$ has points of multiplicity at most $m-1$ (see [Ma]). Though most fashionable today, we will not use this terminology here, since we feel that in the surface case the classical one is equally appropriate.

Then one defines the Kodaira dimension $\kappa(S, C / m)$ of the pair $(S, C / m)$ to be $-\infty$ if $a_{m n}(n C)=0$ for all $n>0$; otherwise, one defines $\kappa(S, C / m)$ to be the dimension of the image of $\phi_{\left|n\left(C+m K_{S}\right)\right|}$, for $n \gg 0$. As we saw, this is a birational invariant.

If $\mathcal{L}$ is a complete planar linear system with no multiple fixed components, and if $f: S \rightarrow \mathbb{P}^{2}$ is a birational morphism such that the general member $C$ of proper transform $\mathcal{L}^{\prime}$ of $\mathcal{L}$ on $S$ is smooth, we can consider $\kappa(S, C)$. This is an invariant of $\mathcal{L}$ by Cremona transformations, which is called the Kodaira dimension of $\mathcal{L}$. We will denote it by $\kappa(\mathcal{L})$. 


\section{$\S 5$. Curves on rational ruled surfaces}

\subsection{Minimal rational surfaces and elementary transformations}

The minimal rational surfaces are $\mathbb{P}^{2}$ and $\mathbb{F}_{n}=\mathbb{P}\left(\mathcal{O}_{\mathbb{P}^{1}} \oplus \mathcal{O}_{\mathbb{P}^{1}}(n)\right), n \geqslant 0$ and $n \neq 1$. The surface $\mathbb{F}_{n}$ is a $\mathbb{P}^{1}$-bundle on $\mathbb{P}^{1}$. We denote by $f_{n}: \mathbb{F}_{n} \rightarrow \mathbb{P}^{1}$ the structure morphism, by $F_{n}$ its general fiber, and by $F_{p}$ the fiber through a point $p \in \mathbb{F}_{n}$. There is a section $E_{n}$ with $E_{n}^{2}=-n$, which is uniquely determined if $n>0$. If $n=0$, then $\left|E_{0}\right|$ is a pencil. We will drop the index $n$ if there is no danger of confusion. Recall that $\operatorname{Pic}\left(\mathbb{F}_{n}\right)$ is generated by the classes of $E$ and $F$.

On $\mathbb{F}_{n}$, we denote by

$$
\mathcal{L}_{n}\left(k, h ;\left[\mu_{1}^{\varepsilon_{1}}, \mu_{2}^{\varepsilon_{2}}, \ldots, \mu_{s}^{\varepsilon_{s}}\right], m_{1}^{e_{1}}, \ldots, m_{r}^{e_{r}}\right)
$$

the linear system of curves in $|k E+h F|$ having $\varepsilon_{i}$ (proper or infinitely near) points of multiplicity at least $\mu_{i}, i=1, \ldots, s$, on $E$ (or on its proper transform) and further $e_{j}$ points of multiplicity at least $m_{j}, i=0, \ldots, r$.

If $\mathcal{L}\left(d ; m_{0}, \ldots, m_{r}\right)$ with $m_{0} \geqslant \cdots \geqslant m_{r}$, we may blow up the base points $p_{0}$ of multiplicity $m_{0}$ and take the proper transform of the system to $\mathbb{F}_{1}$, thus getting the system $\mathcal{L}_{1}\left(d-m_{0}, d ; m_{1}, \ldots, m_{r}\right)$. In this way we will look at planar linear systems as linear systems on $\mathbb{F}_{1}$.

The surfaces $\mathbb{F}_{n}$ can be realized in a projective space as surfaces of minimal degree (see $[\mathrm{dP}]$, $[\mathrm{Con}]$, and $[\mathrm{EH}])$. Consider on $\mathbb{F}_{n}$ the base point free linear system $|E+h F|$, with $h \geqslant n$. If $h=n=0$, then $|E|$ is a pencil. Otherwise, it determines a morphism $\phi=\phi_{|E+h F|}: \mathbb{F}_{n} \rightarrow \mathbb{P}^{r}$, with $r=2 h-n+1$, whose image is a possibly singular surface $\Sigma$ of degree $r-1=2 h-n$. In fact $\phi: \mathbb{F}_{n} \rightarrow \Sigma$ is an isomorphism as soon as $h>n$. In case $h=n>0, \Sigma$ is the cone over a rational normal curve of degree $r-1$, and $\phi$ is an isomorphism off the negative curve $E$, which is contracted to the vertex of the cone. The surface $\Sigma$ is denoted as $S(h-n, h)$, since it is the scroll described by the lines joining corresponding points on two rational normal curves of degree $h-n$ and $h$ in independent spaces. This applies also in the degenerate case $h=n$.

An elementary transformation $\operatorname{elm}_{p}$ centered at a point $p \in \mathbb{F}_{n}$ is the composition of the blowing up of $\mathbb{F}_{n}$ at $p$ and the blowing down of the proper transform of the fiber through $p$, which is a $(-1)$-curve. If $p \notin E$, then $\operatorname{elm}_{p}: \mathbb{F}_{n \rightarrow-\rightarrow} \mathbb{F}_{n-1}$; otherwise, $\operatorname{elm}_{p}: \mathbb{F}_{n}-\rightarrow \mathbb{F}_{n+1}$. 
The composition $\operatorname{elm}_{p_{r}} \circ \cdots \circ \operatorname{elm}_{p_{2}} \circ \operatorname{elm}_{p_{1}}$ of $r$ elementary transformations centered at points $p_{1}, p_{2}, \ldots, p_{r}$ will be denoted by $\operatorname{elm}_{p_{1}, \ldots, p_{r}}$. Only the point $p_{1}$ belongs to $\mathbb{F}_{n}$, whereas $p_{i} \in \operatorname{elm}_{p_{1}, \ldots, p_{i-1}}\left(\mathbb{F}_{n}\right)$, for all $i=2, \ldots, r$.

A birational map $\phi: \mathbb{F}_{n} \rightarrow \mathbb{F}_{n^{\prime}}$ sending the pencil $\left|F_{n}\right|$ to the pencil $\left|F_{n^{\prime}}\right|$, that is, making the following diagram commutative,

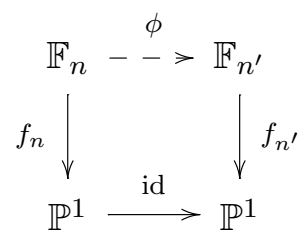

is called a fibered birational map. For example, de Jonquières transformations can be regarded as fibered birational maps $\mathbb{F}_{1} \rightarrow \mathbb{F}_{1}$.

Any fibered birational map is a composition of elementary transformations. This is an aspect of Sarkisov's program in dimension 2 (see [Ma]).

\subsection{Iitaka's $\sharp$-models (sharp models)}

Consider a pair $\left(\mathbb{F}_{n}, \mathcal{L}\right)$, with $\mathcal{L}$ as in $(14)$, and call $m_{1}$ the maximum multiplicity of singular base points of $\mathcal{L}$. We will assume in the sequel that $h \geqslant k n$, so that the curve $E_{n}$ does not split off from the system $\mathcal{L}$. Following Iitaka ([I1], [I2]), we say that the pair $\left(\mathbb{F}_{n}, \mathcal{L}\right)$ is $\sharp$-minimal or a $\sharp$-model (resp., $\sharp \sharp$-minimal, or a $\sharp \sharp$-model) when

- $k \geqslant 2 m_{1}$ (resp., $k>2 m_{1}$ ) if $n \geqslant 2$;

- $k \geqslant 2 m_{1}$ (resp., $k>2 m_{1}$ ) and $h-k \geqslant m_{1}$ if $n=1$;

- $h, k \geqslant 2 m_{1}$ (resp., $h, k>2 m_{1}$ ) if $n=0$.

This is essentially the same as saying that the pair $\left(\mathbb{F}_{n}, 2 C / k\right)$ has canonical (resp., terminal) singularities.

If a pair $\left(\mathbb{F}_{n}, \mathcal{L}\right)$ is not $\sharp$-minimal, one may get a $\sharp$-model by performing elementary transformations based at points of multiplicity $\mu$ with $2 \mu>k$.

In [I1] and [I2], Iitaka described many interesting properties of $\sharp$-models and $\sharp \sharp$-models. In particular, the following result will be useful for us.

TheOREM 5.1 ([I2, Theorem 4, page 316]). One has the following:

(i) if $\left(\mathbb{F}_{n}, \mathcal{L}\right) \sim\left(\mathbb{F}_{n^{\prime}}, \mathcal{L}^{\prime}\right),\left(\mathbb{F}_{n}, \mathcal{L}\right)$ is a $\sharp \sharp$-model, and $\left(\mathbb{F}_{n^{\prime}}, \mathcal{L}^{\prime}\right)$ is a $\sharp$-model, then $\left(\mathbb{F}_{n}, \mathcal{L}\right)$ and $\left(\mathbb{F}_{n^{\prime}}, \mathcal{L}^{\prime}\right)$ are isomorphic;

(ii) if $\left(\mathbb{F}_{n}, \mathcal{L}\right)$, with $\mathcal{L} \subset \mathcal{L}_{n}(k, h)$, and $\left(\mathbb{F}_{n^{\prime}}, \mathcal{L}^{\prime}\right)$ are both $\sharp$-models and are birationally equivalent via a map $\phi$ fitting in a diagram (15), then either $\phi$ is an isomorphism, or $k=2 m$ is even and $\left(\mathbb{F}_{n^{\prime}}, \mathcal{L}^{\prime}\right)$ is obtained 
from $\left(\mathbb{F}_{n}, \mathcal{L}\right)$ with a sequence of elementary transformations based at points of multiplicity $m$. If $\mathcal{L}=\mathcal{L}_{n}\left(k, h ; m_{1}, \ldots, m_{r}\right)$, then $m_{1}, \ldots, m_{r}$ are birational invariants of $\sharp$-models birationally equivalent to $\left(\mathbb{F}_{n}, \mathcal{L}\right)$.

Proof. Part (i) is the uniqueness of terminal models. In any event, set $m=m_{1}$. One has $\operatorname{ad}_{m}(\mathcal{L})=\mathcal{L}_{n}(k-2 m, h-m(n+2))$. This system is very ample on $\mathbb{F}_{n}$, except for $h=k n$, in which case it is very ample on $S(0, n)$. By Remark 4.5, part (i) immediately follows. Part (ii) follows from a similar argument, left to the reader, applied to $\operatorname{ad}_{m-1}(\mathcal{L})$.

\subsection{Definition of $b$-models (flat models) and $\lfloor$-models (natural models)}

Given a pair $\left(\mathbb{F}_{n^{\prime}}, \mathcal{L}^{\prime}\right)$ as above, there is a minimal $n \geqslant 0$ such that $\left(\mathbb{F}_{n}, \mathcal{L}\right)$ is a $\sharp$-model birational to $\left(\mathbb{F}_{n^{\prime}}, \mathcal{L}^{\prime}\right)$. We call it a b-model, and we call $n$ the b-index of $\left(\mathbb{F}_{n^{\prime}}, \mathcal{L}^{\prime}\right)$.

Proposition 5.2. Let $\left(\mathbb{F}_{n}, \mathcal{L}\right)$ be a b-model with $\mathcal{L}$ as in (14). If $n>0$, then

(i) all base points of $\mathcal{L}$ of multiplicity $m \geqslant k / 2$ lie on $E_{n}$;

(ii) if $\left(\mathbb{F}_{n}, \mathcal{L}^{\prime}\right)$ is a b-model birational to $\left(\mathbb{F}_{n}, \mathcal{L}\right)$ via a map $\phi$ fitting in a diagram (15), and either $k$ is odd or $\mathcal{L}$ does not have infinitely near base points of multiplicity $m$ on $E_{n}$, then $\phi$ is an isomorphism.

Proof. By Theorem 5.1, the assertion is clear if $k$ is odd, since in this case $\left(\mathbb{F}_{n}, \mathcal{L}\right)$ is a $\sharp \sharp$-model. So assume that $k$ is even.

Part (i) follows from the very definition of a b-model, since an elementary transformation performed at a base point of multiplicity $k / 2$ off $E_{n}$ would drop $n$ and keep sharpness.

As for part (ii), again by Theorem 5.1, the map $\phi$ is a composition of elementary transformations based at points of multiplicity $m=k / 2$. Each of them preserves sharpness, so this series of maps never involves $\mathbb{F}_{m}$, with $m<$ $n$. On the other hand, by part (i), each elementary transformation creating a point of multiplicity $m$ off the negative curve has to be compensated by another elementary transformation at a point of multiplicity $m$ sending this back on the negative curve. The hypothesis about the points of multiplicity $m$ on $E_{n}$ shows that each of these transformations is the inverse of a previous one.

REMARK 5.3. The assumption that $n>0$ and about the points of multiplicity $m$ on $E_{n}$ in Proposition 5.2 is essential, as one sees by considering linear systems of the form $\mathcal{L}_{0}\left(2 m, h ; m^{e}\right)$, with $h \geqslant n$ and $e \geqslant 2$, and 
$\mathcal{L}_{n}\left(2 m, h ; m^{2}\right)$, with $h \geqslant m n$ and the second point of multiplicity $m$ infinitely near to the first proper point on $E_{n}$, in a direction which is not tangent to the one of $E_{n}$.

Let $\left(\mathbb{F}_{n}, \mathcal{L}\right)$ be a pair with $\mathcal{L}$ as in (14). Again we will assume that $h \geqslant k n$. We say that the pair $\left(\mathbb{F}_{n}, \mathcal{L}\right)$ is $\downarrow$-minimal, or a $\downarrow$-model, when either $n=0$ and the general member of $\mathcal{L}$ is smooth, or $n>0$ and

- $k=2 m+\epsilon, m \geqslant 1, \epsilon=0,1$;

- there is no base point of $\mathcal{L}$ of multiplicity $\mu \geqslant 2 m+\epsilon-1$;

- there is no base point of multiplicity $\mu \geqslant 2$ along $E_{n}$.

A $\downarrow$-model certainly exists, and it is obtained, for example, from a $\sharp$ model by a sequence of elementary transformations based at the singular base points of $\mathcal{L}$ on $E$.

Proposition 5.4. If $\left(\mathbb{F}_{n}, \mathcal{L}\right)$ and $\left(\mathbb{F}_{n^{\prime}}, \mathcal{L}^{\prime}\right)$ are both $\downarrow$-models with positive b-index and are birationally equivalent via a map $\phi$ fitting in a diagram (15), then $\phi$ is an isomorphism.

Proof. Let $\left(\mathbb{F}_{m}, \tilde{\mathcal{L}}\right)$ and $\left(\mathbb{F}_{m^{\prime}}, \tilde{\mathcal{L}}^{\prime}\right)$ be b-models birational to $\left(\mathbb{F}_{n}, \mathcal{L}\right)$ and $\left(\mathbb{F}_{n^{\prime}}, \mathcal{L}^{\prime}\right)$ via fibered birational maps. Then, by Proposition 5.2 and the positivity assumption on the b-index, they are isomorphic. Moreover, $\left(\mathbb{F}_{n}, \mathcal{L}\right)$ and $\left(\mathbb{F}_{n^{\prime}}, \mathcal{L}^{\prime}\right)$ are obtained by this unique model $\left(\mathbb{F}_{m}, \tilde{\mathcal{L}}\right)$ by performing elementary transformations based at the singular base points of $\tilde{\mathcal{L}}$ along $E_{m}$. The assertion follows.

\section{§6. Linear systems of rational curves}

In this section we recall some basic results about complete linear systems of rational curves; in particular, we are interested in a plane birational model with minimal degree. This goes back to the classical Italian school (see [Con] for results and references; for a recent reference, see [I2, page 192]).

Proposition 6.1. Let $S$ be a smooth, irreducible, projective surface with $q(S)=0$, and let $D$ be a nonzero nef divisor with $h^{0}\left(S, \mathcal{O}_{S}\left(D+K_{S}\right)\right)=0$. Then $S$ is rational and either

(i) $D^{2}=0$ and $|D|$ is composed with an irreducible base point free pencil of rational curves; or

(ii) $D^{2}>0$ and $|D|$ is an irreducible base point free linear system of rational curves of dimension $D^{2}+1$. 
Proof. Assume first that $D^{2}=0$. By the Riemann-Roch theorem, one has

$$
0=h^{0}\left(S, \mathcal{O}_{S}\left(D+K_{S}\right)\right) \geqslant 1+D \cdot K_{S} / 2
$$

so $D \cdot K_{S} \leqslant-2$. Again by the Riemann-Roch theorem, one has $h^{0}(S$, $\left.\mathcal{O}_{S}(D)\right) \geqslant 2$. Write now $|D|=F+|M|$, where $M$ is the movable part.

By the nefness property of $D$, we have $F \cdot D=M \cdot D=0$; hence, $F^{2}=$ $M^{2}=-F \cdot M$. If $F \cdot M>0$, then $M^{2}<0$, a contradiction. Since $M$ is nef, we have $F^{2}=M^{2}=F \cdot M=0$. Then $M$ is composed with a base point free irreducible pencil $|L|$ with $p_{a}(L)=0$. By Noether's theorem, $S$ is a rational surface.

We claim that $F=0$. Otherwise, since $L \cdot F=0$, by Zariski's lemma we have two relatively prime positive integers $p, q$ such that $q F \equiv p L$. Since $K \cdot L=-2$ and $K \cdot F$ is even, we have $q=1$; hence, $F \equiv p L$, a contradiction.

Assume now that $d=D^{2}>0$. Since $D$ is nef and big, it is also numerically connected. Since $h^{0}\left(S, \mathcal{O}_{S}\left(D+K_{S}\right)\right)=0$ and $q=0$, one has $p_{a}(D)=$ 0 . By the Riemann-Roch theorem, one has $h^{0}\left(S, \mathcal{O}_{S}(D)\right) \geqslant D^{2}+2$. Let $p_{1}, p_{2}, \ldots, p_{d}$ be general points on $S$, and consider the sublinear system $\mathcal{D} \subset$ $|D|$ consisting of all curves passing through $p_{1}, \ldots, p_{d}$. Blow up $p_{1}, \ldots, p_{d}$, consider the proper transform $\mathcal{D}^{\prime}$ of $\mathcal{D}$ on the blowup $S^{\prime}$, and take a general curve $C$ in $\mathcal{D}^{\prime}$. Then $C^{2}=0$, and one sees that $C$ is still nef and numerically connected.

In the short exact sequence

$$
\begin{aligned}
0 & \rightarrow H^{0}\left(S^{\prime}, \mathcal{O}_{S^{\prime}}\right) \simeq \mathbb{C} \rightarrow H^{0}\left(S^{\prime}, \mathcal{O}_{S^{\prime}}(C)\right) \\
& \rightarrow H^{0}\left(C, \mathcal{O}_{C}(C)\right) \rightarrow 0=H^{1}\left(S^{\prime}, \mathcal{O}_{S^{\prime}}\right)
\end{aligned}
$$

one has $h^{0}\left(S^{\prime}, \mathcal{O}_{S^{\prime}}(C)\right) \geqslant 2$; hence, $h^{0}\left(C, \mathcal{O}_{C}(C)\right) \geqslant 1$. By [CFM, Corollary A.2] one has $\mathcal{O}_{C}(C) \simeq \mathcal{O}_{C}$; therefore, $h^{0}\left(C, \mathcal{O}_{C}(C)\right)=1$, and $|C|=\mathcal{D}^{\prime}$ is a base point free pencil whose general member is irreducible. This implies that $h^{0}\left(S, \mathcal{O}_{S}(D)\right)=D^{2}+2$.

Next we recall the description of Cremona minimal birational models of pairs $(S,|D|)$, with $S$ and $D$ as in Proposition 6.1 .

CASE $D^{2}=0$. As we saw, $|D|$ is composed with a base point free pencil $|L|$ of rational curves.

By blowing down all $(-1)$-cycles $Z$ such that $Z \cdot L=0$, we have a birational morphism $f: S \rightarrow \mathbb{F}_{n}$, for some $n$, which maps $|L|$ to the ruling $|F|$ 
of $\mathbb{F}_{n}$ (one of the rulings if $n=0$ ). By making elementary transformations at general points, we see that

$$
(S,|L|) \sim\left(\mathbb{F}_{n},|F|\right) \sim\left(\mathbb{F}_{1},|F|\right) .
$$

By contracting the $(-1)$-section, this in turn is birationally equivalent to $\left(\mathbb{P}^{2}, \mathcal{L}(1 ; 1)\right)$.

CASE $D^{2}=d>0$. The linear system $|D|$ determines a birational morphism $\varphi: S \rightarrow \Sigma \subseteq \mathbb{P}^{d+1}$, where $\Sigma$ is a surface of degree $d$. According to the del Pezzo classification of minimal degree projective surfaces (see [dP], [Con], and [EH]), we have the following possibilities.

- $\Sigma \simeq \mathbb{P}^{2}$ and $d=1$ or 4 ; accordingly, either $(S,|D|) \sim\left(\mathbb{P}^{2}, \mathcal{L}(1)\right)$ or $(S,|D|) \sim\left(\mathbb{P}^{2}, \mathcal{L}(2)\right)$.

- $d=a+b$, with $0 \leqslant a \leqslant b$, and $\Sigma=S(a, b)$; the minimal resolution of singularities of $\Sigma$ is $\mathbb{F}_{b-a}$ fitting in a diagram

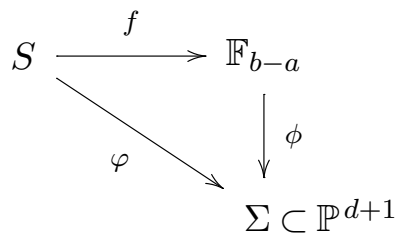

where $f$ is a birational morphism and $\phi$ is determined by the linear system $|E+b F|$, so $(S,|D|) \sim\left(\mathbb{F}_{b-a}, \mathcal{L}_{b-a}(1, b)\right)$.

If $b>a$, make $b-a-1$ elementary transformations based at general points of $\mathbb{F}_{b-a}$. In this way,

$$
(S,|D|) \sim\left(\mathbb{F}_{b-a}, \mathcal{L}_{b-a}(1, b)\right) \sim\left(\mathbb{F}_{1}, \mathcal{L}_{1}\left(1, b ;\left[1^{b-a-1}\right]\right)\right),
$$

where the $b-a-1$ simple base points are general on $E_{1}$. By contracting $E_{1}$, one has

$$
(S,|D|) \sim\left(\mathbb{F}_{1}, \mathcal{L}_{1}\left(1, b ;\left[1^{b-a-1}\right]\right)\right) \sim\left(\mathbb{P}^{2}, \mathcal{L}\left(b ;\left(b-1,\left[1^{b-a-1}\right]\right)\right)\right),
$$

where the $b-a-1$ simple base points are general in the first-order infinitesimal neighborhood of the point of multiplicity $b-1$.

If $b=a$, make one elementary transformation based at a general point of $\mathbb{F}_{0}$, and then contract the $(-1)$-section. In this way,

$$
(S,|D|) \sim\left(\mathbb{F}_{0}, \mathcal{L}(1, b)\right) \sim\left(\mathbb{F}_{1}, \mathcal{L}_{1}(1, b+1 ; 1)\right) \sim\left(\mathbb{P}^{2}, \mathcal{L}(b+1 ; b, 1)\right) .
$$


Now we can state the following result.

Theorem 6.2. Let $(S, D)$ be as in Proposition 6.1. Then $(S,|D|)$ is birationally equivalent to one and only one of the following:

(a) $\left(\mathbb{F}_{1}, \mathcal{L}_{1}(0,1)\right)$;

$\left(b^{j}\right)\left(\mathbb{P}^{2}, \mathcal{L}(j)\right), j=1,2 ;$

$\left(c_{1}^{d}\right)\left(\mathbb{F}_{1}, \mathcal{L}_{1}(1, d)\right), d \geqslant 2 ;$

$\left(c_{0}^{d}\right)\left(\mathbb{F}_{0}, \mathcal{L}_{0}(1, d-1)\right), d \geqslant 2$; or

$\left(c_{n}^{d}\right)\left(\mathbb{F}_{n}, \mathcal{L}_{n}(1, d)\right), 2 \leqslant n \leqslant d$.

These, in turn, are birationally equivalent to the following Cremona minimal pairs $\left(\mathbb{P}^{2}, \mathcal{L}\right)$, where $\mathcal{L}$ is one, and only one, of the following:

(i) $\mathcal{L}(1 ; 1)$, of dimension 1 , corresponding to case $(a)$;

(ii) $\mathcal{L}(j), j=1,2$, of dimension 2 and 5 , respectively, corresponding to case $\left(b^{j}\right)$;

(iii) $\mathcal{L}(d ; d-1), d \geqslant 2$, of dimension $2 d$, corresponding to case $\left(c_{1}^{d}\right)$;

(iv) $\mathcal{L}(d ; d-1,1), d \geqslant 2$, of dimension $2 d-1$, corresponding to case $\left(c_{0}^{d}\right)$; or

(v) $\mathcal{L}\left(d ;\left(d-1,\left[1^{n-1}\right]\right)\right), 2 \leqslant n \leqslant d$, of dimension $2 d-n+1$, with the simple base points infinitely near of order 1 to the point of multiplicity $d-1$, corresponding to case $\left(c_{n}^{d}\right)$.

Proof. Part of the proof is given by [Con], [Na1], and [Na2], with classical methods (cf. [D], which uses Mori's theory). Since we could not find a proper reference for the full statement, we give a proof here.

The birational equivalence to one of the pairs in $(a),\left(b^{j}\right),\left(c_{n}^{d}\right)$ has been proved above (cf. [D]). Pairs $(a),\left(b^{j}\right),\left(c_{0}^{d}\right),\left(c_{1}^{d}\right)$, and $\left(c_{n}^{d}\right), n \geqslant 2$, are, respectively, birationally equivalent to pairs (i), (ii), (iii), (iv), and (v). Their Cremona minimality follows from Lemma 2.1.

Finally, we prove that pairs $\left(\mathbb{P}^{2}, \mathcal{L}\right)$, with $\mathcal{L}$ as in $(\mathrm{i})-(\mathrm{v})$, are birationally distinct. To see this, taking into account the Cremona minimality, it suffices to remark that if these linear systems have the same dimension, then they have different Cremona degrees.

REMARK 6.3. All linear systems in Theorem 6.2 , except $\mathcal{L}(1)$ and $\mathcal{L}(1 ; 1)$, are such that $\operatorname{cdeg}(\mathcal{L})>\operatorname{cdeg}(C)$, where $C \in \mathcal{L}$ is the general curve, since clearly $\operatorname{cdeg}(C)=1$. 


\section{$\S 7$. Admissible plane models and Cremona transformations}

Let $\left(\mathbb{F}_{n}, \mathcal{L}^{\prime}\right)$ be a pair where $n \geqslant 2$ and $\mathcal{L}^{\prime}=\mathcal{L}_{n}\left(k, h ; m_{1}, \ldots\right)$ is not empty, where the indicated multiplicities are the effective ones. By performing $n-1$ elementary transformations at general points $p_{1}, \ldots, p_{n-1}$ of $\mathbb{F}_{n}$, and then by blowing down the (-1)-section $E_{1}$ (cf. Case $D^{2}=d>0$ before Theorem 6.2 ), one gets a plane model $\left(\mathbb{P}^{2}, \mathcal{L}\right)$ with

$$
\mathcal{L}=\mathcal{L}\left(h ;\left(h-k,\left[k^{n-1}\right]\right), m_{1}, \ldots\right) .
$$

It may happen that some of the points of multiplicity $m_{1}, \ldots$ are infinitely near to the point of multiplicity $h-k$. This occurs if and only if some of the base points of $\mathcal{L}^{\prime}$ lie on $E_{n}$.

More generally, one can make the following process. We consider a fibered birational map $\gamma: \mathbb{F}_{n} \rightarrow \mathbb{F}_{1}$ such that $\gamma\left(E_{n}\right)=E_{1}$. In particular, $\gamma$ is a sequence of elementary transformations. By blowing down the $(-1)$-curve $E_{1}$ to a point $p$, one gets a planar linear system $\mathcal{L}$ having multiplicity $\operatorname{deg}(\mathcal{L})-k$ at $p$.

In general, a pair $\left(\mathbb{P}^{2}, \mathcal{L}\right)$ with

$$
\mathcal{L}=\mathcal{L}\left(h ; h-k, \nu_{1}, \ldots\right)
$$

is said to be admissible if all base points $p_{i}$ of $\mathcal{L}$ with multiplicity $\nu_{i}>k / 2$, $i=1, \ldots, r$, are infinitely near to the base point $p$ of multiplicity $h-k$ and if

$$
h-3\left[\frac{k}{2}\right]>\sum_{i=1}^{r} \nu_{i}-r\left[\frac{k}{2}\right] .
$$

This implies that $p$ is the point of maximal multiplicity of $\mathcal{L}$.

Note that $\mathcal{L}$ is admissible if and only if all its multiples

$$
\mathcal{L}\left(i h ; i(h-k), i \nu_{1}, \ldots\right), \quad i \geqslant 1
$$

are admissible.

If $k=2 m$, the $m$-adjoint of $\mathcal{L}$ is

$$
\operatorname{ad}_{m}(\mathcal{L})=\mathcal{L}\left(d ;\left(d,\left\{\mu_{1}, \ldots, \mu_{r}\right\}\right)\right)
$$

where $\mu_{i}=\nu_{i}-m, i=1, \ldots, r$, and $d=\operatorname{deg}(\mathcal{L})-3 m>\sum_{i=1}^{r} \mu_{i}$, which is indeed equivalent to $\left(\mathbb{P}^{2}, \mathcal{L}\right)$ being admissible.

Now we prove the following result. 
THEOREM 7.1. Let $\left(\mathbb{P}^{2}, \mathcal{L}\right)$ be an admissible plane model with $\mathcal{L}$ irreducible. Let $\gamma: \mathbb{P}^{2} \rightarrow \mathbb{P}^{2}$ be a Cremona transformation such that $\operatorname{deg}\left(\gamma_{*}(\mathcal{L})\right) \leqslant \operatorname{deg}(\mathcal{L})$. Then there exists a de Jonquières transformation $\phi:$ $\mathbb{P}^{2} \rightarrow \mathbb{P}^{2}$ such that $\operatorname{deg}\left(\phi_{*}(\mathcal{L})\right) \leqslant \operatorname{deg}\left(\gamma_{*}(\mathcal{L})\right)$.

Beginning of proof. By the Noether-Castelnuovo theorem (Theorem 1.1), $\gamma$ is the composition of finitely many quadratic and linear transformations. We may and will assume that the number of involved quadratic transformations is minimal.

Let $p$ be the maximal multiplicity point of $\mathcal{L}$, and let $p_{i}, i=1, \ldots, r$ be the points of multiplicity

$$
\nu_{i}>m:=\left[\frac{\operatorname{deg}(\mathcal{L})-\operatorname{mult}_{p}(\mathcal{L})}{2}\right],
$$

so $\operatorname{ad}_{m}(\mathcal{L})$ is given by $(16)$. We may assume that $k=2 m$; otherwise, we replace $\mathcal{L}$ with its double.

Let $\Lambda=\mathcal{L}\left(\delta ; \alpha, \alpha_{1}, \ldots, \alpha_{r}, \beta_{1}, \ldots, \beta_{s}\right)$ be the homaloidal net defining $\gamma$, where $\alpha_{i}, i=1, \ldots, r$ ( $\alpha$, resp.) is the multiplicity of $\Lambda$ at $p_{i}$ (at $p$, resp.), and $\beta_{j}, j=1, \ldots, s$ is the multiplicity at the other base points $q_{1}, \ldots, q_{s}$ of $\Lambda$.

The linear system $\operatorname{ad}_{m}(\mathcal{L})$ has $b=\sum_{i=1}^{r} \mu_{i}$ fixed lines, say, $L_{1}, \ldots, L_{t}$, $t \leqslant r$, each $L_{i}$ counted with multiplicity $b_{i}$ with $b=\sum_{j=1}^{t} b_{j}$. The movable part $\mathcal{L}(d-b ; d-b)$ of $\operatorname{ad}_{m}(\mathcal{L})$ is composed with the pencil $\mathcal{L}_{0}=\mathcal{L}(1 ; 1)$ of lines through $p$. Our hypothesis says that $\operatorname{deg}\left(\operatorname{ad}_{m}\left(\gamma_{*}(\mathcal{L})\right)\right) \leqslant \operatorname{ad}_{m}(\mathcal{L})$. This implies that either

(1) $\gamma$ contracts a fixed line to a point, or

(2) $\gamma$ does not contract any fixed line, and therefore $\gamma$ maps $\mathcal{L}_{0}$ to a pencil of lines, in which case $\operatorname{deg}\left(\phi_{*}(\mathcal{L})\right)=\operatorname{deg}(\mathcal{L})$ and $\gamma$ is a de Jonquières. In case (2), there is nothing else to prove. So we may and will assume that case (1) holds and that $\gamma$ is not a de Jonquières. We will then argue by induction on the simplicity of $\gamma$. This ends the first part of the proof.

Next we need the following lemma.

LEMMA 7.2. In the above setting, there is a fixed line of $\operatorname{ad}_{m}(\mathcal{L})$, say $L_{1}$, contracted by $\gamma$, passing through $p$ and an infinitely near point $p_{1}$ to $p$, such that $\delta=\alpha+\alpha_{1}$, with $\alpha \geqslant \alpha_{1} \geqslant \alpha_{i}, \beta_{j}, i=1, \ldots, r, j=1, \ldots, s$.

Proof. We may and will assume that $L_{i}, i=1, \ldots, t$ is the line passing through $p$ and that $p_{i}>^{1} p$. If there is an $i, 1 \leqslant i \leqslant t$, such that $\alpha_{i}=\delta-\alpha$, 
we may assume that $i=1$ and the rest of the assertion follows. Suppose, by contradiction, that $\alpha_{i}<\delta-\alpha$ for each $i=1, \ldots, t$.

The Cremona transformation $\gamma$ maps the pencil $\mathcal{L}_{0}$ to a pencil of curves of degree $\ell=\operatorname{deg}\left(\gamma_{*}\left(\mathcal{L}_{0}\right)\right)=\delta-\alpha \geqslant 2$ and maps the line $L_{i}, i=1, \ldots, t$, to a curve of degree $\ell_{i}=\operatorname{deg}\left(\gamma_{*}\left(L_{i}\right)\right) \leqslant \delta-\alpha-\alpha_{i}$. Then, either $\ell_{i}>0$ or $\ell_{i}=0$, which means that there is at least a proper or infinitely near point $q_{j_{i}}$ on $L_{i}$ or on its proper transform. By (13), one has

$$
\operatorname{deg}\left(\operatorname{ad}_{m}\left(\gamma_{*}(\mathcal{L})\right)\right)=\operatorname{deg}\left(\gamma_{!}\left(\operatorname{ad}_{m}(\mathcal{L})\right)\right)-d \alpha-\sum_{i=1}^{r} \mu_{i} \alpha_{i}+\sum_{j=1}^{s} h_{j} \beta_{j}
$$

where $0 \leqslant h_{j}=m-h_{j}^{\prime}$ where $h_{j}^{\prime}$ is the multiplicity of $\mathcal{L}$ at $q_{j}, j=1, \ldots, s$ and the total transform $\gamma_{!}\left(\operatorname{ad}_{m}(\mathcal{L})\right)$ of $\operatorname{ad}_{m}(\mathcal{L})$ via $\gamma$ has degree

$$
\operatorname{deg}\left(\gamma_{!}\left(\operatorname{ad}_{m}(\mathcal{L})\right)\right)=(d-b) \ell+\sum_{i=1}^{t} b_{i} \ell_{i}+d \alpha+\sum_{i=1}^{t} b_{i} \alpha_{i}+\sum_{i=1}^{t} \sum_{q_{j} \in L_{i}} b_{i} \beta_{j}
$$

where the last sum runs over the proper or infinitely near base points $q_{j}$ of $\Lambda$ which belong to the proper transform of $L_{i}$. (There is no $p_{i}, i=t+$ $1, \ldots, r$, among such points; otherwise, $L_{i}$ should be a fixed component of L.) Therefore, $(17)$ and (18) imply that

$$
\begin{aligned}
\operatorname{deg}\left(\operatorname{ad}_{m}\left(\phi_{*}(\mathcal{L})\right)\right) & \geqslant(d-b) \ell+\sum_{i=1}^{t} b_{i}\left(\ell_{i}+\sum_{q_{j} \in L_{i}} b_{i} \beta_{j}\right) \\
& \geqslant 2 d-2 b+\sum_{i=1}^{t} b_{i}=d+(d-b)>d,
\end{aligned}
$$

which contradicts the assumption that $\operatorname{deg}\left(\gamma_{*}(\mathcal{L})\right) \leqslant \operatorname{deg}(\mathcal{L})$.

Proof of Theorem 7.1 (continued). By Lemma 7.2, the simplicity of $\gamma$, that is, of $\Lambda$, is $\left(k_{\Lambda}, h_{\Lambda}, s_{\Lambda}\right)$, where $k_{\Lambda}=\delta-\alpha=\alpha_{1}>1$. By the proof of the Noether-Castelnuovo theorem (Theorem 1.1) in Appendix A, there is a quadratic transformation $\psi: \mathbb{P}^{2} \rightarrow \mathbb{P}^{2}$ centered at $p$ such that $\gamma=\gamma^{\prime} \circ$ $\psi$, with $\gamma^{\prime}$ simpler than $\gamma$. Set $\mathcal{L}^{\prime}=\psi_{*}(\mathcal{L})$. If $\operatorname{deg}\left(\mathcal{L}^{\prime}\right) \leqslant \operatorname{deg}(\mathcal{L})$, then the minimality assumption on $\gamma$ implies that $\gamma$ is quadratic, a contradiction because we are assuming that $\gamma$ is not a de Jonquières. Therefore, $\operatorname{deg}\left(\mathcal{L}^{\prime}\right)>$ $\operatorname{deg}(\mathcal{L}) \geqslant \operatorname{deg}\left(\gamma_{*}(\mathcal{L})\right)=\operatorname{deg}\left(\gamma_{*}^{\prime}\left(\mathcal{L}^{\prime}\right)\right)$. Furthermore, the number of quadratic 
transformations involved in the factorization of $\gamma^{\prime}$ is minimal, as well as the analogous number for $\gamma$.

In Lemma 7.3 below, we prove that we may choose the quadratic transformation $\psi$ in such a way that $\operatorname{ad}_{m}\left(\mathcal{L}^{\prime}\right)=\mathcal{L}\left(d^{\prime} ;\left(d^{\prime},\left\{\mu_{1}^{\prime}, \ldots, \mu_{r}^{\prime}\right\}\right)\right)$ and that $\mathcal{L}^{\prime}$ has maximal multiplicity at $p^{\prime}$; namely, $\psi$ maps the pencil $\mathcal{L}_{0}$ of lines through $p$ to the pencil of lines through $p^{\prime}$. By induction, there is a de Jonquières transformation $\varphi$ centered at $p^{\prime}$ such that $\operatorname{deg}\left(\varphi_{*}\left(\mathcal{L}^{\prime}\right)\right) \leqslant$ $\operatorname{deg}\left(\gamma_{*}^{\prime}\left(\mathcal{L}^{\prime}\right)\right)=\operatorname{deg}\left(\gamma_{*}(\mathcal{L})\right)$. Therefore, $\phi:=\varphi \circ \psi$ is a de Jonquières transformation centered at $p$ such that $\operatorname{deg}\left(\phi_{*}(\mathcal{L})\right) \leqslant \operatorname{deg}\left(\gamma_{*}(\mathcal{L})\right)$, which concludes the proof.

LEMMA 7.3. In the above setting, we may choose the quadratic transformation $\psi$ in such a way that $\left(\mathbb{P}^{2}, \mathcal{L}^{\prime}\right)$ is admissible; namely, $\operatorname{ad}_{m}\left(\mathcal{L}^{\prime}\right)=$ $\mathcal{L}\left(d^{\prime} ;\left(d^{\prime},\left\{\mu_{1}^{\prime}, \ldots, \mu_{r}^{\prime}\right\}\right)\right)$.

Proof. Since $p_{1}$ is a point of multiplicity $\alpha_{1}>k_{\Lambda} / 2$, the proof of NoetherCastelnuovo's theorem in Appendix A implies that there is a quadratic transformation $\psi$ based at $p$, at $q>^{1} p$, and at $x$, which lowers the simplicity of $\Lambda$. Let $\mathcal{L}^{\prime}=\psi_{*}(\mathcal{L})$, which has maximal multiplicity at $p^{\prime}$, the point corresponding to $p$ via $\psi$. We have to prove that $\mathcal{L}^{\prime}$ does not have base points of multiplicity $\mu>m$ off $p^{\prime}$.

Assume first that $x$ is a proper point; hence, the multiplicity of $\mathcal{L}$ at $x$ is $\nu \leqslant m$. Then a point of multiplicity $\mu>m$ for $\mathcal{L}^{\prime}$ off $p^{\prime}$ could come only from a point $y>^{1} q, y \emptyset p$. But in that case, the quadratic transformation based at $p, q, y$ lowers the degree of $\mathcal{L}$, a contradiction.

The alternative is that $x>^{1} q$. As above, the multiplicity of $\mathcal{L}$ at $x$ is $\nu \leqslant$ $m$, and therefore $\psi$ cannot produce points of multiplicity $\mu>m$ off $p^{\prime}$.

\section{$\S 8$. Weight of a curve on an $\mathbb{F}_{n}$ and good plane models}

Here we introduce the notions needed for finding Cremona minimal pairs.

Let $\left(\mathbb{F}_{n}, C\right)$ be a pair where $n \geqslant 2$ and $C$ is a singular, irreducible curve, and let $p \notin E$ be a singular point of $C$. The weighted oriented tree, or briefly tree, $T_{p}$ of $p \in C \subset \mathbb{F}_{n}$ is defined as follows.

Step 1. The root $v_{p}$ of $T_{p}$ corresponds to $p$ with weight the multiplicity of $C$ at $p$.

Step 2. Make elm $\operatorname{lm}_{p}$, and let $q=\operatorname{elm}_{p}\left(F_{p}\right) \in E$. Consider the proper transform $C^{\prime}$ of $C$ and the singular points $p_{1}, \ldots, p_{r}$ of $C^{\prime}$ lying on $F_{q}$ off $E$; then $T_{p}$ has one vertex $v_{p_{i}}$, with weight the multiplicity of $C^{\prime}$ at $p_{i}$, and one arrow $v_{p} \rightarrow v_{p_{i}}$, for each $i=1, \ldots, r$. 
Step 3. Iterate Step 2 for $p_{1}, \ldots, p_{r}$ until either no further singular point of $C^{\prime}$ shows up or the maximal length of oriented chains in the tree is $n-1$.

The weighted oriented forest, or briefly forest, $G=G\left(\mathbb{F}_{n}, C\right)$ of the pair $\left(\mathbb{F}_{n}, C\right)$ is constructed as follows.

- If $F_{p}$ is the fiber of the ruling $|F|$ containing a singular point $p$ of $C$ off $E$, then $G$ has a vertex $v_{F_{p}}$, with zero weight.

- For each singular point $p \notin E$ of $C, G$ then contains the tree $T_{p}$ of $p \in C$, connected to $v_{F_{p}}$ with an arrow $v_{F_{p}} \rightarrow v_{p}$.

- $G$ has further vertices $v_{p_{i}}$, with weight 1 , and $v_{F_{p_{i}}}$, with zero weight, and an arrow $v_{F_{p_{i}}} \rightarrow v_{p_{i}}$, for each $i=1, \ldots, n-1$, where $p_{1}, \ldots, p_{n-1}$ are general points of $C$.

A path $P$ in $G$ is the union of oriented chains in distinct connected components of $G$, each chain starting from a vertex of weight 0 . The length of $P$ is the number of arrows that it contains. Given a path of length $n-1$, the set $\left\{p_{1}, \ldots, p_{n-1}\right\}$ of corresponding points is called a cluster for the pair $\left(\mathbb{F}_{n}, C\right)$. The weight of $P$ is the sum of the weights of all vertices where $P$ is supported. A path $P$ is called good if $P$ has length $n-1$ and maximal weight. Accordingly, the cluster $\left\{p_{1}, \ldots, p_{n-1}\right\}$ of corresponding points is called good. Setting $m_{i}, i=1, \ldots, n-1$, the multiplicity of $C$ at the infinitely near or proper point $p_{i}$, which is the weight of $G$ at the corresponding vertex, we may and will assume that $m_{1} \geqslant m_{2} \geqslant \cdots \geqslant m_{n-1} \geqslant 1$, and we say that $m_{1}, \ldots, m_{n-1}$ is a good sequence of multiplicities for the pair $\left(\mathbb{F}_{n}, C\right)$. Accordingly, the plane model $\left(\mathbb{P}^{2}, B\right)$, obtained by performing the elementary transformations at $p_{1}, \ldots, p_{n-1}$ and then blowing down the $(-1)$-curve $E_{1} \subset \mathbb{F}_{1}$, is called good. The weight $w\left(\mathbb{F}_{n}, C\right)$ of the pair $\left(\mathbb{F}_{n}, C\right)$ is the weight $w(P)=\sum_{i=1}^{n-1} m_{i}$ of a good path $P$ in the forest $G$.

REMARK 8.1. The number of connected components of $G=G\left(\mathbb{F}_{n}, C\right)$ equals the number of fibers of $|F|$ containing singular points of $C$ off $E$, increased by $n-1$. On each connected component of $G$ there is a unique vertex of weight 0 .

There exist good paths in $G$. For example, if $n=2$, a good path is just an arrow $v_{F_{p}} \rightarrow v_{p}$ where $p$ is a point of maximal multiplicity of $C$ off $E_{2}$.

Example 8.2. (a) Let $\left(\mathbb{F}_{n}, C\right)$ be a pair with $n \geqslant 2$ and $C$ smooth. Then $1^{n-1}$ is the unique good sequence of multiplicities of $\left(\mathbb{F}_{n}, C\right)$, and any set of $n-1$ general points of $C$ is a good cluster. 
(b) Let $\left(\mathbb{F}_{n}, C\right)$ be a pair with $n \geqslant 2$ and $C$ irreducible. Suppose that the largest $n-1$ multiplicities $m_{1} \geqslant m_{2} \geqslant \cdots \geqslant m_{n-1}$ of $C$ can be found, respectively, at points $p_{1}, \ldots, p_{n-1}$ on distinct fibers, off $E_{n}$. Then $w\left(\mathbb{F}_{n}, C\right)=$ $\sum_{i=1}^{n-1} m_{i}$, and $m_{1}, \ldots, m_{n-1}$ is a good sequence of multiplicities of $C$. In particular, if the singularities of $C$ are only at proper points on distinct fibers, off $E_{n}$, then there is a unique good sequence of multiplicities of $C$, but perhaps different good clusters.

(c) Let $\left(\mathbb{F}_{3}, C\right), C \in \mathcal{L}_{3}(6,18 ; 3,(2,[2]))$; that is, $C$ has a triple point $p$ and a tacnode $p^{\prime}$. Suppose that $p, p^{\prime}$ lie on the same fiber $F_{p}=F_{p^{\prime}}$ off $E_{3}$ and that the tacnodal tangent at $p^{\prime}$ is different from the tangent line to $F_{p}$ at $p^{\prime}$. Then the weighted oriented forest $G=G\left(\mathbb{F}_{3}, C\right)$ of $\left(\mathbb{F}_{3}, C\right)$ is

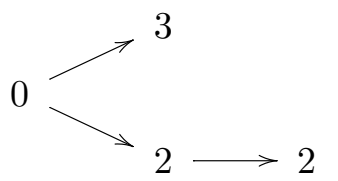

$$
0 \longrightarrow 1 \quad 0 \longrightarrow 1
$$

that has two different good sequences of multiplicities 2,2 and 3,1 , determined by the different good paths: $0 \rightarrow 2 \rightarrow 2$ and $\{0 \rightarrow 3\} \cup\{0 \rightarrow 1\}$.

Accordingly, one gets two birationally equivalent different Cremona minimal pairs $\left(\mathbb{P}^{2}, B_{1}\right)$ and $\left(\mathbb{P}^{2}, B_{2}\right)$ with $B_{1} \in \mathcal{L}\left(14 ;\left(8,\left[4^{2}\right]\right), 3\right)$ and $B_{2} \in \mathcal{L}(14 ;$ $\left.(8,[5,3]), 2^{2}\right)$, where all singularities of $B_{1}, B_{2}$ are infinitely near to the point of multiplicity 8 , and their configuration is described, respectively, by the following two diagrams
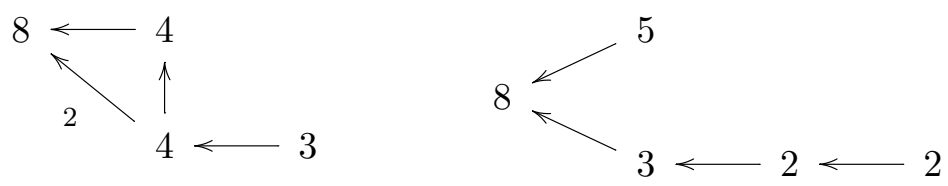

where $m_{1} \stackrel{r}{\longrightarrow} m_{2}$ means that the point of multiplicity $m_{1}$ is proximate and infinitely near of order $r$ to the point of multiplicity $m_{2}$ (no $r$ means $r=1$ ).

This example shows that a good sequence of multiplicities may well be not unique.

Now we study the properties of good sequences of multiplicities of $\natural$ models obtained from $\sharp$-models.

REMARK 8.3. (i) Let $\left(\mathbb{F}_{n^{\prime}}, C^{\prime}\right)$ be a b-model where $n^{\prime} \geqslant 1, C^{\prime} \in \mathcal{L}_{n^{\prime}}\left(2 m, h^{\prime}\right.$; $\left.m_{1}, \ldots\right)$, and $C^{\prime}$ is irreducible. The $\natural$-model $\left(\mathbb{F}_{n}, C\right)$ fibered birationally 
equivalent to $\left(\mathbb{F}_{n^{\prime}}, C^{\prime}\right)$ is such that $C$ has at most $n-n^{\prime}$ proper or infinitely near points of multiplicity $\mu>m$.

(ii) Let $m_{1}^{\prime} \geqslant \cdots \geqslant m_{n-1}^{\prime}=\bar{m}$ be a good sequence of multiplicities for the ६-model $\left(\mathbb{F}_{n}, C\right)$ as above, and let $\left\{p_{1}, \ldots, p_{n-1}\right\}$ be the corresponding good cluster.

The proper transform $C_{2}=\left(\operatorname{elm}_{p_{1}, \ldots, p_{n-2}}\right)_{*}(C) \subset \mathbb{F}_{2}$ has multiplicity $\bar{m}$ at $p_{n-1} \notin E_{2}$. The definition of good cluster implies that $C_{2}$ has no point of multiplicity $\mu>\bar{m}$ off $E_{2}$. If $\left(\mathbb{P}^{2}, B\right)$ is the good model of $\left(\mathbb{F}_{n}, C\right)$ and if $q \in \mathbb{P}^{2}$ is the point of $B$ with the highest multiplicity, it follows that each point of $B$ with multiplicity $\mu>\bar{m}$, if any, is infinitely near to $q$.

(iii) If $\bar{m} \geqslant m$, then $C$ has multiplicity $\mu \geqslant m$ at each one of the $n-$ 1 points $p_{1}, \ldots, p_{n-1}$ of the good cluster; hence, $n^{\prime}=1$ by (i), and $C_{1}=$ $\left(\operatorname{elm}_{p_{1}, \ldots, p_{n-1}}\right)_{*}(C)=C^{\prime}$ on $\mathbb{F}_{1}$. Thus $B=\sigma_{*}\left(C_{1}\right) \in \mathcal{L}\left(h^{\prime} ; h^{\prime}-2 m, m_{1}, \ldots\right)$, with $m_{1} \leqslant m$, and the good plane model $\left(\mathbb{P}^{2}, B\right)$ is Cremona minimal by Theorem 2.3. In particular, if $n^{\prime} \geqslant 2$, it follows that $\bar{m}<m$.

(iv) Suppose that $B$ has multiplicity $m_{1}>m$ at a point $q_{1}>^{1} q$, and let $L_{1}$ be the line passing through $q$ and $q_{1}$. If $B$ meets $L_{1}$ at a proper or infinitely near point $q^{\prime}$, different from $q$ and $q_{1}$, then $B$ has multiplicity $\mu<m$ at $q^{\prime}$, because

$$
\operatorname{deg}(B)-\operatorname{mult}_{q}(B)-\operatorname{mult}_{q_{1}}(B)=2 m-m_{1}<m .
$$

Theorem 8.4. Let $\left(\mathbb{P}^{2}, B\right)$ be a good model of a $\downarrow$-model $\left(\mathbb{F}_{n}, C\right), n \geqslant 2$, with b-index at least $2, C$ irreducible, $C \in \mathcal{L}_{n}\left(2 m+\epsilon, h ; m_{1}, \ldots\right)$, and $\epsilon=0,1$. Then the pair $\left(\mathbb{P}^{2}, B\right)$ is Cremona minimal.

Proof. Let $p_{0}$ be the point of maximal multiplicity of $B$. Suppose that there is a Cremona transformation $\gamma$ such that $\operatorname{deg}\left(\gamma_{*}(B)\right)<\operatorname{deg}(B)$ and $\operatorname{deg}\left(\gamma_{*}(B)\right)$ is minimum. Since $\left(\mathbb{P}^{2}, B\right)$ is admissible, by Theorem 7.1 there is a de Jonquières transformation $\phi$ centered at $p_{0}$ such that $\operatorname{deg}\left(\phi_{*}(B)\right)=$ $\operatorname{deg}\left(\gamma_{*}(B)\right)<\operatorname{deg}(B)$, and, moreover, $\phi$ maps the pencil of lines through the maximal multiplicity point of $B$ to the pencil of lines through the maximal multiplicity point of $B^{\prime}=\phi_{*}(B)$.

The curve $B$ sits in a linear system of the form $\mathcal{L}(d+3 m ;(d+m-$ $\left.\left.\epsilon,\left[2 m+\epsilon-\mu_{1}, \ldots, 2 m+\epsilon-\mu_{n-1}\right], m_{1}^{\prime}, \ldots\right)\right)$, where $d=\operatorname{deg}\left(\operatorname{ad}_{m}(B)\right)$, the Ł-model is $\left(\mathbb{F}_{n}, C\right)$, and $\mu_{1}, \ldots, \mu_{n-1}$ is a good sequence of multiplicities of $C$ (not necessarily in decreasing order). Let $\Lambda=\mathcal{L}\left(\delta ; \delta-1,1^{2 \delta-2}\right.$ ) be the homaloidal net defining $\phi$. Let $\ell \leqslant n-1$ be the number of points among those of multiplicities $2 m+\epsilon-\mu_{1}, \ldots, 2 m+\epsilon-\mu_{n-1}$ for $B$, which are simple 
base points for $\Lambda$. By proximity, we have $\ell \leqslant \delta-1$. Then

$$
\operatorname{deg}\left(\phi_{*}(B)\right)=\delta(d+3 m)-(\delta-1)(d+m-\epsilon)-\sum_{i=1}^{\ell}\left(2 m+\epsilon-\mu_{i}\right)-\sum_{\ell+1}^{2 \delta-2} \mu_{j}^{\prime},
$$

where $\mu_{j}^{\prime}$ are the multiplicities of $B$ at the remaining base points of $\Lambda$. Therefore, one has

$\operatorname{deg}\left(\phi_{*}(B)\right)=2 m(\delta-\ell)+d+m+\epsilon(\delta-1-\ell)+\sum_{i=1}^{\ell} \mu_{i}-\sum_{i=\ell+1}^{2 \ell} \mu_{i}^{\prime}-\sum_{i=2 \ell+1}^{2 \delta-2} \mu_{i}^{\prime}$,

where we assume that the $\mu_{i}^{\prime}$ are ordered in decreasing order for $\ell+1 \leqslant i \leqslant$ $2 \delta-2$. Note that the general curve of the proper transform of $\Lambda$ on $\mathbb{F}_{n}$ is a smooth section. Hence, all the base points of this proper transform are parts of paths in the relevant forest. Therefore, $\sum_{i=1}^{\ell} \mu_{i} \geqslant \sum_{i=\ell+1}^{2 \ell} \mu_{i}^{\prime}$. Moreover, we have $\mu_{i}^{\prime} \leqslant m$ for $2 \ell+1 \leqslant i \leqslant 2 \delta-2$ as a consequence of the assumption on the $b$-index (see Remark 8.3(iii)). Hence, we deduce

$$
\operatorname{deg}\left(\phi_{*}(B)\right) \geqslant 2 m(\delta-\ell)+d+m-2(\delta-\ell-1) m=d+3 m,
$$

a contradiction.

REMARK 8.5. The above proof shows that one may have $\operatorname{deg}\left(\phi_{*}(B)\right)=$ $\operatorname{deg}(B)$ for a de Jonquières transformation only if $\sum_{i=1}^{l} \mu_{i}=\sum_{i=\ell+1}^{2 \ell} \mu_{i}^{\prime}$, $\mu_{i}^{\prime}=m$ for $2 \ell+1 \leqslant i \leqslant 2 \delta-2$ and $\ell=\delta-1$ if $\epsilon=1$.

\section{§. The main classification theorem}

Let $(S, C)$ be a pair. We will say that it presents the line case if it is birationally equivalent to $\left(\mathbb{P}^{2}, L\right)$, where $L$ is a line. For example, if $C$ is part of a $(-1)$-cycle on $S$, then $(S, C)$ presents the line case. Coolidge [Coo] stated the following theorem, which gives necessary and sufficient conditions for a pair $(S, C)$ to present the line case.

Theorem 9.1 (Coolidge [Coo]). Let $B$ be an irreducible plane curve. Then $\left(\mathbb{P}^{2}, B\right)$ presents the line case if and only if $\operatorname{ad}_{m}(B)=\emptyset$, for all $m>0$.

Unfortunately, Coolidge's proof ([Coo, pages 396-398]; written again in [KM, pages 772-773]) is incomplete. Kumar and Murthy gave a correct proof of Theorem 9.1 with different methods. They actually proved more (see [KM, Theorem 2.1 and Corollary 2.4]). 
Theorem 9.2 (Kumar-Murthy $[\mathrm{KM}]$ ). Let $B$ be an irreducible plane curve. Then $\left(\mathbb{P}^{2}, B\right)$ presents the line case if and only if $\kappa\left(\mathbb{P}^{2}, B\right)=-\infty$, which is equivalent to $\operatorname{ad}_{1}(B)=\operatorname{ad}_{2}(B)=\emptyset$. In particular, if $C$ is the minimal desingularization of $B$ on a surface and $C^{2}=-n$, then $\left(\mathbb{P}^{2}, B\right) \sim$ $\left(\mathbb{F}_{n}, E\right)$.

Remark 9.3. In the above setting, if $C$ is rational and $C^{2} \geqslant-3$, then $\left|C+K_{S}\right|=\left|C+2 K_{S}\right|=\emptyset$ and $(S, C)$ presents the line case. Hence, if $C$ is irreducible and the pair $(S, C)$ does not present the line case, then $C$ verifies the hypothesis of Proposition 4.1(ii).

Next we prove a birational classification theorem for all pairs $(S, C)$, with $S$ rational, producing for each of them a unique model on a minimal rational surface or on $\mathbb{F}_{1}$. Moreover, we produce for each pair a plane model of minimal Cremona degree.

First, we introduce some additional notation. Given a pair $(S, C)$, with $S$ smooth and rational and $C$ smooth, not presenting the line case, let $m:=$ $m(S, C)$ be the minimum positive integer $m$ such that $\left|C+m K_{S}\right| \neq \emptyset$ and $\left|C+(m+1) K_{S}\right|=\emptyset$ and

$$
\alpha:=\alpha(S, C)=\operatorname{dim}\left(\left|C+m K_{S}\right|\right)=h^{0}\left(S, \mathcal{O}_{S}\left(C+m K_{S}\right)\right)-1 .
$$

By the results in Section $4.2, m$ and $\alpha$ are birational invariants of the pair $(S, C)$. Note that, if $C$ is rational, then $m \geqslant 2$ and, moreover, $C$ is not part of a $(-1)$-cycle on $S$.

Our main results are the following theorems.

Theorem 9.4 (Birational classification of pairs). Let $(S, C)$ be a pair with $S$ rational and $C$ smooth and irreducible, and suppose that $(S, C)$ does not present the line case. Let $m=m(S, C)$, and let $\alpha=\alpha(S, C)$. Then $(S, C)$ is birationally equivalent to one of the following pairs:

$\left(d p_{1}\right)\left(\mathbb{P}^{2}, D\right)$, where $D \in \mathcal{L}\left(3 m ; m_{0}, \ldots\right)$, with $m_{0} \leqslant m$, and $\alpha=0$;

$\left(d p_{2}\right)\left(\mathbb{F}_{n}, D\right)$, where $D \in \mathcal{L}_{n}\left(2 m,(2+n) m ; m_{1}, \ldots\right)$, with $m_{1}<m, n=$ 0,2 , and $\alpha=0$;

$(r)\left(\mathbb{F}_{n}, D\right), 0 \leqslant n \leqslant 2+[\alpha / m]$, where $D \in \mathcal{L}_{n}\left(2 m,(2+n) m+\alpha ; m_{1}, \ldots\right)$ is b-minimal, that is, $m_{1} \leqslant m, \alpha>0$, and, if $n \geqslant 1$, all singular points of multiplicity $m$ are on $E_{n}$;

$\left(b_{1}\right)\left(\mathbb{P}^{2}, D\right)$, where $D \in \mathcal{L}\left(3 m+[\alpha / 2] ; m_{0}, \ldots\right)$, with $m_{0} \leqslant m, \alpha=2,5$;

$\left(b_{2}\right)\left(\mathbb{F}_{n}, D\right)$, where $D \in \mathcal{L}_{n}\left(2 m+1,(2+n) m+(\alpha+n-1) / 2 ; m_{1}, \ldots\right)$ is b-minimal, with $m_{1} \leqslant m, \alpha+n \equiv 1,(\bmod 2)$, and $\alpha \geqslant 3+n$ if $0 \leqslant n \leqslant 1$, whereas $\alpha \geqslant 3+(n-2)(2 m+1) \geqslant 3$ if $n \geqslant 2$. 
The above pairs may be birationally equivalent and not isomorphic only if we are in one of the following cases:

(i) $\left(d p_{1}\right)$, with $m_{0}=m_{1}=m_{2}=m$; or

(ii) $(r)$, with $n=0$ and $D$ having at least two points of multiplicity $m$.

REMARK 9.5. By taking into account the proof of Proposition 5.2, one sees that case (ii) can be improved. Indeed, $D$ may have more points of multiplicity $m$, provided that performing elementary transformations there forces us to make the inverse transformations to go back to the b-model. This can be expressed in terms of clusters on the $q$-model, but we do not dwell on this here.

REMARK 9.6. All pairs in the statement of Theorem 9.4 except types $\left(d p_{1}\right)$ and $\left(b_{1}\right)$ are $\sharp$-models (see $\left.\S 5.1\right)$. By blowing up a point of multiplicity $m_{0}$, one sees that they are, respectively, birationally equivalent to the $\sharp$ models:

$$
\left(\mathbb{F}_{1}, D^{\prime}\right), \quad D^{\prime} \in \mathcal{L}_{1}\left(3 m-m_{0}, 3 m ; m_{1}, \ldots\right),
$$

with $m_{1} \leqslant m_{0} \leqslant m$,

$$
\left(\mathbb{F}_{1}, D^{\prime}\right), \quad D^{\prime} \in \mathcal{L}_{1}\left(3 m-m_{0}+[\alpha / 2], 3 m+[\alpha / 2] ; m_{1}, \ldots\right),
$$

Pairs $\left(d p_{2}\right)$ and $(r)$ if either $n=0$ or $m>m_{1}$, pair $\left(b_{1}\right)$, and pair (20) are $\sharp \sharp$-models. Pair (19) is a $\sharp \sharp$-model if either $m_{0}<m$ or $m=m_{0}>m_{1}$. Pair $(r)$ with $n \geqslant 1$ has a positive $b$-index.

In cases $(r)$ and $\left(b_{2}\right)$, with $n \geqslant 2$, since the $b$-index is positive, we may consider the unique $\downarrow$-model $\left(\mathbb{F}_{n+v}, D_{\natural}\right)$ which is fibered birationally equivalent to $\left(\mathbb{F}_{n}, D\right)$ (see Proposition 5.4). Let $q_{1}, \ldots, q_{v}$ be the proper or infinitely near singular points of $D$ lying on $E_{n}$ or on its strict transform, and let $\mu_{1}, \ldots, \mu_{v}$ be the respective multiplicities, where we may assume that $\mu_{1} \geqslant$ $\cdots \geqslant \mu_{v} \geqslant 2$. Set $\gamma=\sum_{i=1}^{v}\left(2 m-\mu_{i}\right)$, and set $n^{\prime}=n+v$. Then $\left(\mathbb{F}_{n+v}, D_{\natural}\right)$ is obtained by performing elementary transformations at $q_{1}, \ldots, q_{v}$, and therefore either $D_{\natural} \in \mathcal{L}_{n^{\prime}}(2 m,(2+n) m+\alpha+\gamma)$, in case $(r)$ with $n \geqslant 1$, or $D_{\natural} \in \mathcal{L}_{n^{\prime}}(2 m+1,(2+n) m+(\alpha+n-1) / 2+\gamma)$, in case $\left(b_{2}\right)$ with $n \geqslant 1$.

Let $m_{1}^{\prime}, m_{2}^{\prime}, \ldots, m_{n^{\prime}-1}^{\prime}$ be a good sequence of multiplicities of the $\downarrow$-model $\left(\mathbb{F}_{n^{\prime}}, D_{\natural}\right)$, and set $\beta=\sum_{i=1}^{n^{\prime}-1} m_{i}^{\prime}$. With this notation, we have the following. 
Theorem 9.7 (Cremona minimal pairs). Let $(S, C)$ be a pair with $S$ rational and $C$ smooth and irreducible, and suppose that $(S, C)$ does not present the line case. Then $(S, C)$ is birationally equivalent to a Cremona minimal pair $\left(\mathbb{P}^{2}, B\right)$, where $B$ belongs to one of the following planar linear systems (where $m$ and $\alpha$ are the birational invariants introduced above):

$\left(c d p_{1}\right) \mathcal{L}\left(3 m ; m_{0}, \ldots\right)$, with $m_{0} \leqslant m$

$\left(c d p_{2}\right) \mathcal{L}\left(4 m-m_{1} ; 2 m-m_{1}, 2 m-m_{1}, m_{2}, \ldots\right)$, with $m_{2} \leqslant m_{1}<m, m_{1}>$ 0

$\left(c d p_{3}\right) \mathcal{L}\left(4 m-m_{1} ; 2 m-m_{1},\left[2 m-m_{1}\right], m_{2}, \ldots\right)$, with $m_{2} \leqslant m_{1}<m, m_{1}>$ 0 ;

$\left(c r_{0}\right) \mathcal{L}\left(4 m-m_{1}+\alpha ; 2 m-m_{1}+\alpha, 2 m-m_{1}, m_{2}, \ldots\right)$, with $m_{2} \leqslant m_{1}<m$, $m_{1}>0$, and $\alpha>0$;

$\left(c r_{1}\right) \mathcal{L}\left(3 m+\alpha ; m+\alpha, m_{1}, \ldots\right)$, with $m_{1} \leqslant m$ and $\alpha>0 ;$

$\left(c r_{2}\right) \mathcal{L}\left((2+n) m-\beta+\alpha+\gamma ;\left(n m-\beta+\alpha+\gamma,\left[2 m-m_{n^{\prime}-1}^{\prime}, 2 m-m_{n^{\prime}-2}^{\prime}\right.\right.\right.$, $\left.\left.\left.\ldots, 2 m-m_{1}^{\prime}\right]\right), m_{n^{\prime}+1}, \ldots\right)$, with $2 \leqslant n \leqslant 2+[\alpha / m], \alpha>0, m \geqslant m_{n^{\prime}+1} \geqslant \ldots$ and $n^{\prime}, \gamma, m_{1}^{\prime}, m_{2}^{\prime}, \ldots, m_{n^{\prime}-1}^{\prime}$, and $\beta$ as above;

$\left(c b_{1}\right) \mathcal{L}\left(3 m+[\alpha / 2] ; m_{0}, \ldots\right)$, with $m_{0} \leqslant m$ and $\alpha=2,5$;

$\left(c b_{2}\right) \mathcal{L}\left(3 m+\alpha / 2 ; m-1+\alpha / 2, m_{1}, \ldots\right)$, with $m_{1} \leqslant m$ and $\alpha \geqslant 4$ is even;

$\left(c b_{3}\right) \mathcal{L}\left(4 m-m_{1}+(\alpha+1) / 2 ; 2 m-m_{1}+(\alpha-1) / 2,2 m+1-m_{1}, m_{2}, \ldots\right)$, with $m_{2} \leqslant m_{1} \leqslant m$ and $\alpha \geqslant 3$ is odd;

$\left(c b_{4}\right) \mathcal{L}((2+n) m-\beta+\gamma+(\alpha+n-1) / 2 ; n m-\beta+\gamma+(\alpha+n-3) / 2$, $\left.\left[2 m+1-m_{n^{\prime}-1}^{\prime}, 2 m+1-m_{n^{\prime}-2}^{\prime}, \ldots, 2 m+1-m_{1}^{\prime}\right], m_{n^{\prime}+1}, \ldots\right)$, with $2 \leqslant n \leqslant$ $2+(\alpha-3) /(2 m+1), m_{n^{\prime}+1} \leqslant m$, and $n^{\prime}, \gamma, m_{1}^{\prime}, m_{2}^{\prime}, \ldots, m_{n^{\prime}-1}^{\prime}$, and $\beta$ as above.

The above pairs may be Cremona, but not projectively, equivalent only if we are in one of the following cases:

(1) $\left(c d p_{1}\right)$ with $m_{0}=m_{1}=m_{2}=m$ and $\left(c_{1}\right)$ with $m_{1}=m_{2}=m$;

(2) $\left(c d p_{2}\right),\left(c d p_{3}\right)$, and $\left(c r_{0}\right)$ with $m_{1}=m_{2}$;

(3) $\left(\mathrm{cr}_{2}\right)$, for different good sequences of multiplicities of the same $\square$ model;

(4) $\left(c b_{4}\right)$, for different good sequences of multiplicities of the same $\square$ model.

Except in cases (3) and (4), the Cremona type of the minimal Cremona pairs in a Cremona equivalence class is unique.

REMARK 9.8. All systems in Theorem 9.7 except $\left(c r_{2}\right)$ and $\left(c b_{4}\right)$ are of Noether type, and therefore, by Theorem 2.3, their Cremona minimality is clear. 
The proof of Theorems 9.4 and 9.7 will follow from the analysis of three different cases, according to the behavior of the nef part of $C+m K_{S}$. Indeed, by Propositions 4.1 and 4.2 , one has $C+m K_{S} \equiv P+N$, where $P$ is the nef and $N$ is the negative part of $C+m K_{S}$ as in (11). Then there are three possible cases:

(A) $P=0$;

(B) $P>0$ and $P^{2}=0$; or

(C) $P>0$ and $P^{2}>0$.

We call cases (A), (B), and (C), respectively, the del Pezzo, ruled, and big cases, and we discuss them separately.

\subsection{The del Pezzo case}

This case is covered by the following.

Proposition 9.9. Let $(S, C)$ be a pair presenting the del Pezzo case. Then $(S, C)$ is birationally equivalent to one and only one of the pairs $\left(d p_{1}\right)$, $\left(d p_{2}\right)$.

Accordingly, $(S, C)$ is birationally equivalent to a Cremona minimal pair $\left(\mathbb{P}^{2}, B\right), B \in \mathcal{L}$, where $\mathcal{L}$ is one and only one of types $\left(c d p_{1}\right),\left(c d p_{2}\right)$, and $\left(c d p_{3}\right)$ in Theorem 9.7.

Proof. Let $f: S \rightarrow S^{\prime}$ be the birational morphism which first blows down the negative part $N$ of $\left|C+m K_{S}\right|$ and then other $(-1)$-cycles in such a way that $S^{\prime}$ is minimal. On $S^{\prime}$, one has $C^{\prime}=f_{*}(C) \equiv-m K_{S^{\prime}}$, and a $(-1)$-cycle $\theta$ on $S$, which is blown down by $f$ and is not part of $N$, is such that $C \cdot \theta=m$. If $S^{\prime}=\mathbb{F}_{n}$, one has $0 \leqslant C^{\prime} \cdot E_{n}=(2-n) m$; therefore, $n \leqslant 2$. Thus, there are three subcases: $S^{\prime}=\mathbb{P}^{2}$, or $S^{\prime}=\mathbb{F}_{0}$, or $S^{\prime}=\mathbb{F}_{2}$.

We may and will assume that, if $S^{\prime}=\mathbb{F}_{0}$ or $\mathbb{F}_{2}$, the birational morphism $S \rightarrow S^{\prime}$ does not factor through a birational morphism $g: S \rightarrow \mathbb{F}_{1}$ which blows down $N$. Indeed, if such a morphism $g$ exists, we may assume that $S^{\prime}=\mathbb{P}^{2}$.

We discuss separately the three cases.

- If $S^{\prime}=\mathbb{P}^{2}$, then $C^{\prime} \equiv 3 m L$, where $L$ is a line; that is, $C^{\prime}$ is a curve of degree $3 m$ with points of multiplicity at most $m$. Hence, we are in cases $\left(d p_{1}\right)$ and $\left(c d p_{1}\right)$.

- If $S^{\prime}=\mathbb{F}_{0}$, then $C^{\prime} \equiv 2 m E_{0}+2 m F_{0}$ and $C^{\prime}$ has points of multiplicity at most $m$. If $C^{\prime}$ had a point $p$ of multiplicity $m$, then $\operatorname{elm}_{p} \circ f: S \rightarrow \mathbb{F}_{1}$ would be a birational morphism which factors through the blowing down of $N$, a contradiction. Therefore, we are in case $\left(d p_{2}\right)$ with $n=0$. Next, 
choose a point $p$ of maximal multiplicity $m_{1}$ of $C^{\prime}$. Perform an elementary transformation elm ${ }_{p}$, and then blow down the (-1)-section $E_{1}$. The result is a pair $\left(c d p_{2}\right)$.

- If $S^{\prime}=\mathbb{F}_{2}$, then $C^{\prime} \equiv 2 m E_{2}+4 m F_{2}$ and $C^{\prime}$ has points of multiplicity at most $m$. Note that $C^{\prime} \cdot E_{2}=0$ implies that $C \cap E_{2}=\emptyset$. The same arguments as above imply that we are in cases $\left(d p_{2}\right), n=2$, and $\left(c d p_{3}\right)$.

The Cremona minimality of the pairs $\left(c d p_{1}\right),\left(c d p_{2}\right)$, and $\left(c d p_{3}\right)$ follows from Theorem 2.3.

Cremona minimality implies that the pairs $\left(c d p_{1}\right)$ are not Cremona equivalent to either $\left(c d p_{2}\right)$ or $\left(c d p_{3}\right)$. To prove that $\left(c d p_{2}\right)$ and $\left(c d p_{3}\right)$ are not Cremona equivalent, note that the image of $\mathbb{P}^{2}$ via the linear system $\operatorname{ad}_{m_{1}}(\mathcal{L})$ is projectively different in the two cases (see Remark 4.5).

The assertions Theorem 9.4(i) and Theorem 9.7(1),(2) regarding the del Pezzo pairs follow from Corollary 2.4.

REMARK 9.10. (i) From the irreducibility of $C$, it follows that the number of points of multiplicity $m$ of $\mathcal{L}$ in case $\left(c d p_{1}\right)$ is at most 9 , if $m>2$ (at most 10 if $m=2$ ).

(ii) An alternative proof of the birational inequivalence of pairs $\left(d p_{1}\right)$ and $\left(d p_{2}\right)$ follows from Iitaka's theorem (Theorem 5.1$)$; since types $\left(d p_{2}\right)$ and $\left(d p_{3}\right)$ are $\sharp \sharp$-minimal and since type $\left(d p_{1}\right)$, considered on $\mathbb{F}_{1}$ as in Remark 9.6, is $\sharp$-minimal.

\subsection{The ruled case}

We deal with this case in the following proposition.

Proposition 9.11. Let $(S, C)$ be a pair presenting the ruled case. Then $(S, C)$ is birationally equivalent to one and only one of the pairs in $(r)$, with the usual exception for $n=0$.

Accordingly, $(S, C)$ is birationally equivalent to a Cremona minimal pair $\left(\mathbb{P}^{2}, B\right), B \in \mathcal{L}$, where $\mathcal{L}$ is one and only one of types $\left(c r_{0}\right),\left(c r_{1}\right)$, and $\left(c r_{2}\right)$.

Proof. Since $\operatorname{ad}_{m+1}(C)=\emptyset$, one has $\left|P+K_{S}\right|=\emptyset$, and Proposition 6.1 implies that $P$ is composed with an irreducible base point free pencil $|L|$ of rational curves, namely, $P \equiv \alpha L$.

Blowing down of all $(-1)$-cycles $Z$ such that $Z \cdot L=0$ gives a birational morphism $f: S \rightarrow \mathbb{F}_{n}$, which maps $|L|$ to the ruling $|F|$ of $\mathbb{F}_{n}$ (one of the rulings if $n=0)$. Therefore,

$$
D=f_{*}(C) \equiv-m K_{\mathbb{F}_{n}}+\alpha F \equiv 2 m E+(m(2+n)+\alpha) F,
$$


and $D$ has points of multiplicity at most $m$. So this is a $\sharp$-model. We may actually assume that $n$ is the b-index of the pair.

Note that $0 \leqslant D \cdot E_{n}=2 m-m n+\alpha$ implies that $n \leqslant 2+\alpha / m$.

If $n=0$, we find case $(r)$ with $n=0$. Then we get $\left(c r_{0}\right)$ from $(r)$ by choosing a point $p \in D$ of maximal multiplicity $m_{1}$, performing the elementary transformation $\operatorname{elm}_{p}$, and then contracting the (-1)-section $E_{1}$.

If $n=1$, we find case $(r)$ with $n=1$. Then we get $\left(c r_{1}\right)$ by contracting the curve $E_{1}$.

Assume now that $n>1$. We are still in case $(r), n \geqslant 2$, and we get type $\left(c r_{2}\right)$ as a good model obtained from the $\downarrow$-model of $\left(\mathbb{F}_{n}, D\right)$ (see Section 5.3).

The birational uniqueness of types in $(r)$, except for those with $n=0$ and at least two points of multiplicity $m$, follows from Remark 9.6, Theorem 5.1, and Proposition 5.2.

As for Cremona minimality, in cases $\left(c r_{i}\right), 0 \leqslant i \leqslant 1$, one applies Theorem 2.3. In case $\left(c r_{2}\right)$, Cremona minimality follows from Theorem 8.4.

The types $\left(c r_{i}\right), 0 \leqslant i \leqslant 1$, are clearly not Cremona equivalent. The types $\left(c r_{2}\right)$ are not Cremona equivalent to the others, since such a Cremona equivalence would induce a fibered birational equivalence between the corresponding b-models (see Theorem 7.1), which would therefore be isomorphic by Theorem 5.2 , a contradiction.

Theorem 9.4(ii) follows from Proposition 5.2 in case $n>0$. If $n=0$ and there is only one point of multiplicity $m$, the assertion follows from Corollary 2.4, applied to case $\left(c r_{0}\right)$. Theorem $9.7(1),(2)$ regarding the ruled pairs follow again from Corollary 2.4. As for Theorem 9.7(3), it follows from Theorem 8.4 and Remark 8.5.

REMARK 9.12. Iitaka's theorem (Theorem 5.1) gives an alternative proof of the fact that pairs of type $(r)$ are birationally inequivalent when $m_{1}<m$, because in that case type $(r)$ is $\sharp \sharp$-minimal.

\subsection{The big case}

Finally, we dispose of the big case. This finishes the analysis of the various possible cases and the proof of Theorems 9.4 and 9.7.

Proposition 9.13. Let $(S, C)$ be a pair presenting the big case. Then $(S, C)$ is birationally equivalent to one and only one of pairs $\left(b_{1}\right)$.

Accordingly, $(S, C)$ is birationally equivalent to a Cremona minimal pair $\left(\mathbb{P}^{2}, B\right), B \in \mathcal{L}$, where $\mathcal{L}$ is one and only one of types $\left(c b_{1}\right),\left(c b_{2}\right),\left(c b_{3}\right)$, and $\left(c b_{4}\right)$ in Theorem 9.7. 
Proof. Since $\left|C+(m+1) K_{S}\right|=\emptyset$, one has $\left|P+K_{S}\right|=\emptyset$, and Proposition 6.1 implies that $|P|$ is a base point free linear system of dimension $\alpha=P^{2}+1>1$. The same argument used after Proposition 6.1 shows that there is a birational morphism $f: S \rightarrow S^{\prime}$, with either $S^{\prime}=\mathbb{P}^{2}$ or $S^{\prime}=\mathbb{F}_{n}$, mapping $|P|$ to a linear system $\left|P^{\prime}\right|=f_{*}(|P|)$, which is one of the cases $\left(b^{j}\right)$, $\left(c_{n}^{d}\right), n \geqslant 0$, of Theorem 6.2. Furthermore, as in the del Pezzo case and the ruled case, the curve $C^{\prime}=f_{*}(C)$ has points of multiplicity at most $m$. In the $\mathbb{F}_{n}$-case we may assume that $n$ is the b-index.

We analyze the different cases which may occur.

$\left(b^{j}\right)$ On $\mathbb{P}^{2}$ one has $\left|P^{\prime}\right|=\mathcal{L}(j)$, so either $\alpha=2$, if $j=1$, or $\alpha=5$, if $j=2$. Hence, $C^{\prime} \equiv-m K_{\mathbb{P}^{2}}+P^{\prime} \equiv(3 m+[\alpha / 2]) L, L$ a line, and we are in case $\left(b_{1}\right)$.

$\left(c_{1}^{d}\right)$ On $\mathbb{F}_{1}$, one has $\left|P^{\prime}\right|=\mathcal{L}_{1}(1, d), d \geqslant 2$, where $d=\alpha / 2$. Hence, $\alpha \geqslant 4$ is even, and $C^{\prime} \equiv-m K_{\mathbb{F}_{1}}+P^{\prime} \equiv(2 m+1) E+(3 m+\alpha / 2) F$, that is, case $\left(b_{2}\right), n=1$.

$\left(c_{0}^{d}\right)$ On $\mathbb{F}_{0}$, one has $\left|P^{\prime}\right|=\mathcal{L}_{0}(1, d-1), d \geqslant 2$, where $d=(\alpha+1) / 2$; thus $\alpha \geqslant 3$ is odd, and $C^{\prime} \equiv-m K_{\mathbb{F}_{0}}+P^{\prime} \equiv(2 m+1) E+(2 m+(\alpha-1) / 2) F$. Hence, we are in case $\left(b_{2}\right), n=0$.

$\left(c_{n}^{d}\right)$ On $\mathbb{F}_{n}, n \geqslant 2$, one has $\left|P^{\prime}\right|=\mathcal{L}_{n}(1, d), d \geqslant n$, where $d=(\alpha+n-1) / 2$, and therefore $C^{\prime} \equiv(2 m+1) E+(m(2+n)+(\alpha+n-1) / 2) F$. Since $0 \leqslant$ $C^{\prime} \cdot E_{n}=m(2-n)+(\alpha-n-1) / 2$, one has $\alpha \geqslant 3+(2 m+1)(n-2)$, and we are in case $\left(b_{2}\right), n \geqslant 2$.

The above pairs are birationally distinct by Theorem 5.1.

Now we deal with Cremona models. Cases $\left(c b_{1}\right)$ and $\left(c b_{2}\right)$ correspond to cases $\left(b_{1}\right)$ and $\left(b_{2}\right), n=1$. In case $\left(b_{2}\right), n=0$, choose a point $p_{1}$ of the highest multiplicity $m_{1}$ of $C^{\prime}$, and perform the elementary transformation $\operatorname{elm}_{p_{1}}: \mathbb{F}_{0} \rightarrow \mathbb{F}_{1}$; by contracting the $(-1)$-curve $E_{1}$ of $\mathbb{F}_{1}$, we arrive at case $\left(c b_{3}\right)$. In case $\left(b_{2}\right), n \geqslant 2$, consider the $q$-model obtained from the $b$ model we have reached (see notation introduced in Section 5.3 and used in Section 9.2), then go to a good plane model, thus getting case $\left(c b_{4}\right)$.

The Cremona minimality of types $\left(c b_{1}\right),\left(c b_{2}\right)$, and $\left(c b_{3}\right)$ follows from Theorem 2.3. That of type $\left(c b_{4}\right)$ can be proved with the same arguments we used in the ruled case for $\left(c r_{2}\right)$. The birational inequivalence of the four cases is clear.

Theorem 9.7(4) follows from Theorem 8.4 and Remark 8.5.

REMARK 9.14. (i) Iitaka's theorem gives an alternative proof of the birationally inequivalence of types $\left(b_{1}\right),\left(b_{2}\right)$, because all of them are $\sharp \sharp$-minimal (considering $\left(b_{1}\right)$ as a pair $(20)$ on $\mathbb{F}_{1}$; see Remark 9.6). 
(ii) From Theorem 6.2 we have that $\left|C+h K_{S}\right|=\emptyset$ for any $h \geqslant m$. By definition, $\left|C+h K_{S}\right| \neq \emptyset$ for $0 \leqslant h \leqslant m$, unless $C$ is rational, in which case $\left|C+K_{S}\right|=\emptyset$.

(iii) The above results can be easily extended to pairs $(S, \mathcal{L})$ with $\mathcal{L}$ a positive dimensional, irreducible linear system on a rational surface $S$. Once one arrives at a $\downarrow$-model, the definition of a good sequence of multiplicities has to be slightly changed, inasmuch as one may perform elementary transformations only at base points of the system or at general points of the surface. We do not further dwell on this here.

\section{$\S 10$. Applications}

\subsection{Minimality of pairs}

Let $(S, C)$ be a pair as usual. The following definitions are due to Iitaka [I1]. The pair $(S, C)$ is called relatively minimal if there is no $(-1)$ curve $E$ on $S$ such that $C \cdot E \leqslant 1$. The pair is called minimal if whenever we have a pair $\left(S^{\prime}, C^{\prime}\right)$ and a birational map $\phi: S^{\prime} \rightarrow S$ inducing an equivalence $(S, C) \sim\left(S^{\prime}, C^{\prime}\right)$, then $\phi$ is a morphism.

Proposition 10.1 (Iitaka [I1]). Let $(S, C)$ be a relatively minimal pair, with $S$ rational and $C$ irreducible. Assume that $\kappa(S, C) \geqslant 0$, that either $m(S, C)>1$ or $m(S, C)=1$, that and $(S, C)$ does not present the ruled case. Then $(S, C)$ is minimal.

Proof. Let $\left(S^{\prime}, C^{\prime}\right)$ be another pair, and let $\phi: S^{\prime} \rightarrow S$ be a birational map inducing an equivalence $(S, C) \sim\left(S^{\prime}, C^{\prime}\right)$.

If $m(S, C)>1$, apply Proposition 4.4 for $m=2$. We have the diagram

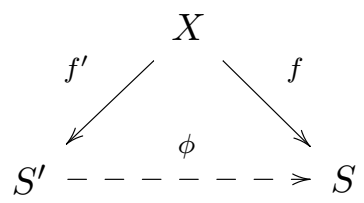

Let $\Theta$ be an $f^{\prime}$-exceptional $(-1)$-cycle on $X$ which is noncontracted by $f$. Then $f_{*}(\Theta)=\theta$ is a $(-1)$-cycle on $S$ such that $C \cdot \theta \leqslant 1$, a contradiction. This proves the assertion in this case.

If $m(S, C)=1$, then we consider $P=C+K_{S}$, which is nef. In the del Pezzo case, we have $C \equiv-K_{S}$, and relative minimality implies that there is no $(-1)$-curve on $S$, proving the assertion. In the big case, note that relative minimality implies that there is no $(-1)$-curve $E$ such that $P \cdot E=0$. 
Then $|P|$ determines a morphism $f: S \rightarrow \Sigma \subset \mathbb{P}^{r}$, with $\Sigma$ a minimal degree surface. Then $S$ is minimal, proving the assertion in this case, too.

Corollary 10.2 (Iitaka [I1]). Let $(S, C)$ be a relatively minimal pair, with $S$ rational and $C$ irreducible. If $\kappa(S, C) \geqslant 0$, then $C^{2}$ and $K_{S}^{2}$ are birational invariant of the pair $(S, C)$.

Proof. The assertion follows from Proposition 10.1 if either $m(S, C)>1$ or $m(S, C)=1$ and $(S, C)$ does not present the ruled case. In the former case, however, we have $C+K_{S} \equiv \alpha L$, where $L$ is a pencil of rational curves. But the relative minimality hypothesis implies that there is no $(-1)$-curve $E$ such that $E \cdot L=0$. Hence, $S$ is a $\mathbb{F}_{n}$, and $C \equiv \alpha L-K_{S}$; thus $C^{2}$ and $K_{S}^{2}$ do not depend on $n$.

\subsection{Low Kodaira dimension}

Proposition 10.3 (Kumar-Murthy [KM], Iitaka [I1]). Let $(S, C)$ be a relatively minimal pair with $S$ rational and $C$ irreducible. Assume that $\kappa=$ $\kappa(S, C)=0,1$. Then $(S, C)$ is one of the following:

(i) $(S, C)$ is the minimal resolution of singularities of $\left(\mathbb{P}^{2}, D\right)$, with $D \in$ $\mathcal{L}\left(3 m ; m^{9}\right), m \geqslant 1 \quad(\kappa=0)$;

(ii) $(S, C)$ is the minimal resolution of singularities of $\left(\mathbb{F}_{n}, D\right), n=0,2$, with $D \in \mathcal{L}\left(2 m,(2+n) m ; m^{8}\right), m \geqslant 1 \quad(\kappa=0)$;

(iii) $(S, C)$ is the minimal resolution of singularities of $\left(\mathbb{P}^{2}, D\right)$, with $D \in$ $\mathcal{L}\left(3 m ; m^{9}, 2\right), m \geqslant 1 \quad(\kappa=0)$;

(iv) $(S, C)$ is the minimal resolution of singularities of $\left(\mathbb{F}_{n}, D\right), n=0,2$, with $D \in \mathcal{L}\left(2 m,(2+n) m ; m^{8}, 2\right), m \geqslant 1 \quad(\kappa=0)$;

(v) $\left(\mathbb{F}_{n}, D\right)$ as in case $(r)$ of Theorem 9.4, with $m=1(\kappa=1)$.

Proof. Let $m=m(S, C)$, and let $m K_{S}+C=P+N$, with $P$ the nef and $N$ the negative part. One has $P^{2}=0$. If we are in the del Pezzo case, then we are in cases (i)-(iv). If we are in the ruled case, since $C+P$ is big, one has $m=1$, and we are in case $(\mathrm{v})$.

Remark 10.4. Proposition 10.3(i),(ii) are birational to minimal Cremona plane curves $D \in \mathcal{L}\left(3 m ; m^{9}\right)$ which, for $m \geqslant 1$, give rise to Halphen's pencils of elliptic curves. Cases (iii) and (iv) are birational plane curves $D \in \mathcal{L}\left(3 m ; m^{9}, 2\right)$ with minimal Cremona degree, which are nodal curves in Halphen's pencils. 


\subsection{Genus 0}

Let $(S, C)$ be a relatively minimal pair with $S$ rational and $C$ irreducible and rational. If $C^{2} \geqslant-3$, then $h^{0}\left(S, \mathcal{O}_{S}\left(2 K_{S}+C\right)\right)=0$ and $(S, C)$ presents the line case.

Proposition 10.5 (Matsuda $[\mathrm{M}])$. Let $(S, C)$ be a relatively minimal pair with $S$ rational and $C$ irreducible and rational. If $C^{2}=-4$ and $(S, C)$ does not present the line case, then $\kappa(S, C)=0$ and $(S, C)$ is as in Proposition 10.3(iii),(iv). The minimal Cremona type of $C$ is $\left(3 m ; m^{9}, 2\right)$, that is, $C$ is a nodal curve in a Halphen's pencil.

Proof. Set $K_{S}^{2}=k$, and set $P=2 K_{S}+C$, which is effective and nef. One has $P^{2}=4(k+1) \geqslant 0$; thus $k \geqslant-1$. Also, $P \cdot K_{S}=2(k+1)$. The RiemannRoch theorem says that $\operatorname{dim}(|P|) \geqslant k+1$. But since $C \cdot P=0$, one has $\operatorname{dim}(|P|)=0$; hence, $k=-1$ and $P^{2}=P \cdot K_{S}=0$. Moreover, $2\left(K_{S}+C\right)=$ $P+C$. This implies that $\kappa(S, C) \leqslant 1$. The assertion follows from Proposition 10.3 .

\subsection{Genus 1}

The following results are classical (see, e.g., [Na1], [Na2]).

Proposition 10.6. Let $(S, C)$ be a relatively minimal pair with $S$ rational and $C$ nef of genus 1. Assume that $C^{2} \geqslant 0$ with $C$ irreducible if $C^{2}=0$. Then $(S, C)$ is as in Proposition 10.3(i),(ii), with $m=m(S, C)=1$ if and only if $C^{2}>0$. In particular, $(S, C)$ is birational to a pair in Proposition 10.3(i).

Proof. Set $K_{S}+C=P$ which is effective and nef. Since $P \cdot C=0$, if $C^{2}>$ 0 , the index theorem yields $P=0$. If $C^{2}=0$, then $P^{2}=0$ and $\kappa(S, C)<2$; thus the assertion follows from Proposition 10.3.

Corollary 10.7. Let $(S, \mathcal{L})$ be a relatively minimal pair with $S$ rational and $\mathcal{L}$ a linear system whose general curve $C$ is nef and irreducible if $C^{2}=0$ and has arithmetic genus 1 and $r=\operatorname{dim}(\mathcal{L}) \geqslant 1$. Then

(i) $(S, \mathcal{L})$ is birational to $\left(\mathbb{P}^{2}, \mathcal{L}\left(3 ; 1^{9-r}\right)\right)$, with $2 \leqslant r \leqslant 9$;

(ii) $(S, \mathcal{L})$ is birational to $\left(\mathbb{P}^{2}, \mathcal{L}\left(3 m ; m^{9}\right)\right)$, with $r=1$; or

(iii) $(S, \mathcal{L})$ is birational to $\left(\mathbb{F}_{n}, \mathcal{L}(2,2+n)\right), n=0,2$, or to $\left(\mathbb{P}^{2}, \mathcal{L}\left(4,2^{2}\right)\right)$, where the two base points may be infinitely near $(r=8)$.

We add the following corollaries. 
Corollary 10.8. Let $(S, C)$ be a pair, with $S$ a rational surface and $C$ an irreducible curve of genus 1 . Then $(S, C)$ is birational to $\left(\mathbb{P}^{2}, B\right)$ with $B$ a smooth plane cubic if and only if $m(S, C)=1$.

Proof. One implication is clear. As for the other, let $P=K_{S}+C$, which we may assume to be nef by contracting all $(-1)$-curves $E$ with $C \cdot E=$ 0. Since $P \cdot C=0$, only the del Pezzo case is possible, and the assertion follows.

Corollary 10.9. Let $(S, C)$ be a relatively minimal pair, with $S$ a rational surface and $C$ an irreducible curve of genus 1 with $C^{2}<0$. Then $C^{2}<$ -1 .

Proof. Assume by contradiction that $C^{2}=-1$. Set $P=K_{S}+C$, which is effective and nef. The case $m(S, C)=1$ is impossible since $P \cdot C=0$. Then $P^{\prime}=2 K_{S}+C$ is effective and nef. Set $k=K_{S}^{2}$. Since $P^{2}=k+1$, we have $k \geqslant-1$. On the other hand, $P^{\prime} \cdot C=1$, and the Riemann-Roch theorem says that $\operatorname{dim}\left(\left|P^{\prime}\right|\right) \geqslant k+1$. This implies that $k=-1$. But then $\left(P^{\prime}\right)^{2}=-1$, a contradiction.

\subsection{De Franchis's theorem}

In this section we deal with the genus 2 case (for a classical reference, see [DF]; however, this is affected, as well as all papers on the subject that appeared before 1901, by the criticism raised by C. Segre to Noether's original proof of the Noether-Castelnuovo theorem [S]; cf. [Ne]).

Proposition 10.10. Let $(S, \mathcal{L})$ be a relatively minimal pair with $S$ rational and $\mathcal{L}$ a complete linear system of curves of arithmetic genus 2 with $r=\operatorname{dim}(\mathcal{L}) \geqslant 1$ whose general curve $C$ is nef. Then $(S, \mathcal{L})$ is as follows:

(i) $\left(\mathbb{F}_{n}, \mathcal{L}_{n}(2,3+n)\right)$, with $0 \leqslant n \leqslant 3$;

(ii) $(S, \mathcal{L})$ is the minimal desingularization of a pair of the form $\left(\mathbb{P}^{2}, \mathcal{L}(6 ;\right.$ $\left.2^{8}\right)$ );

(iii) $(S, \mathcal{L})$ is the minimal desingularization of a pair of the form $\left(\mathbb{P}^{2}, \mathcal{L}(7 ;\right.$ $\left.\left.3,2^{10}\right)\right)$;

(iv) $(S, \mathcal{L})$ is the minimal desingularization of a pair of the form $\left(\mathbb{P}^{2}, \mathcal{L}(9 ;\right.$ $\left.\left.3^{8}, 2^{2}\right)\right)$;

$(\mathrm{v})(S, \mathcal{L})$ is the minimal desingularization of a pair of the form $\left(\mathbb{P}^{2}\right.$, $\left.\mathcal{L}\left(13 ; 5,4^{9}\right)\right)$.

Proof. Set $K_{S}+C=P$, which is effective and nef. Since $P \cdot C=2$, then $P \neq 0$. Actually, $|P|$ is a pencil. 
If $P^{2}=0$, then $P \cdot K_{S}=-2$, and therefore $|P|$ is a pencil of curves of genus 0 and there is no $(-1)$-curve $E$ such that $E \cdot P=0$, namely, $S=\mathbb{F}_{n}$, for some $n \geqslant 0$. Hence, $m=m(S, C)=1$ and $\alpha=\alpha(S, C)=1$, and we are in case $(r)$ of Theorem 9.4, which leads to case (i).

If $P^{2}>0$, the index theorem yields $C^{2} \leqslant 4$. If $P^{2}=1$, then $P \cdot K_{S}=-1$. By contracting all $(-1)$-curves $E$ such that $P \cdot E=1$, we obtain a new pair $\left(S^{\prime}, P^{\prime}\right)$. This may let $\mathcal{L}$ acquire base points of multiplicity 2 . Then we may apply Proposition 10.6, which tells us what $\left(S^{\prime}, P^{\prime}\right)$ is, and we conclude that $(S, P)$ is the minimal desingularization of a pair of the form $\left(\mathbb{P}^{2}, \mathcal{L}\left(6 ; 2^{8}\right)\right)$ or $\left(\mathbb{F}_{n}, \mathcal{L}_{n}\left(4,2(2+n) ; 2^{7}\right)\right)$, with $n=0,2$. Thus we are in case (ii). In particular, this applies when $C^{2}=4$.

So we are left with the case $P^{2} \geqslant 2$, and the cases $C^{2}=1, P^{2}=4$, and $C^{2}=P^{2}=2$ can be excluded by the index theorem.

Consider first the case $r>1$. Set $K_{S}^{2}=k$. If $-K_{S}$ is not effective, then $\mathcal{L}$ cuts on $P$ a linear series of dimension 2 and degree 2. Hence, $P$ is rational; then $m(S, C)=1$, and we are in the big case since $P^{2}>0$. This contradicts $\alpha=1$. So $-K_{S}>0$. Then $2 \leqslant P^{2}=P \cdot\left(K_{S}+C\right) \leqslant P \cdot C=2$. Hence, $P^{2}=2$ and $P \cdot K_{S}=0$. Moreover, $C^{2} \leqslant 1, C \cdot K_{S}=2-C^{2}$, so that $0=P \cdot K_{S}=$ $k+2-C^{2}$. In conclusion, $C \cdot K_{S}=-k+K_{S} \cdot P=-k=2-C^{2} \geqslant 0$, a contradiction.

So we may assume that $r=1$. Then $C^{2} \leqslant 2$, since $1=r=h^{0}\left(S, \mathcal{O}_{S}(C)\right)-$ $1=h^{0}\left(C, \mathcal{O}_{C}(C)\right) \geqslant C^{2}-1$.

Let $C^{2}=c, C \cdot K_{S}=2-c$, with $0 \leqslant c \leqslant 1$. Then $P^{2}=k+4-c, P \cdot K_{S}=$ $k+2-c$; hence, $p_{a}(P)=k+4-c=P^{2}$. Set $P^{\prime}=P+K_{S}=C+2 K_{S}$. Then $\operatorname{dim}\left(\left|P^{\prime}\right|\right)=P^{2}-1$ and $C \cdot P^{\prime}=4-c$. Therefore, $P^{2} \leqslant 3-c$, since $P^{\prime}-C \equiv 2 K_{S}$ is not effective.

So we are left with the cases $C^{2}=0,2 \leqslant P^{2} \leqslant 3, C^{2}=1$, and $P^{2}=2$.

If $C^{2}=0, P^{2}=2$, then $k=-2, P^{\prime}$ is still nef, and $\left(P^{\prime}\right)^{2}=0$. Moreover, $P \cdot P^{\prime}=2$ implies that $\left|P^{\prime}\right|$ is a pencil of rational curves, so we are in the ruled case with $m=2, \alpha=1$, and $(S, \mathcal{L})$ is the minimal desingularization of a pair of the form $\left(\mathbb{F}_{n}, \mathcal{L}_{n}\left(4,5+2 n ; 2^{10}\right)\right)$, with $0 \leqslant n \leqslant 2$, and we are in case (iii).

If $C^{2}=1, P^{2}=2$, then $k=-1, P^{\prime}$ is nef, and $\left(P^{\prime}\right)^{2}=1, P^{\prime} \cdot K_{S}=-1$. Hence, $\left|P^{\prime}\right|$ is a pencil of curves of arithmetic genus 1. By Corollary 10.7, we are in case (iv).

If $C^{2}=0, P^{2}=3$, then $k=-1, P^{\prime}$ is nef, and $\left(P^{\prime}\right)^{2}=4, P^{\prime} \cdot K_{S}=0$. Now let us contract all $(-1)$-curves $E$ such that $E \cdot P^{\prime}=0$, producing a new pair $\left(S_{1}, P_{1}\right)$ on which $\mathcal{L}$ may acquire base points of multiplicity 2 . Set 
$P_{1}^{\prime}=K_{S_{1}}+P_{1}$, which is nef, and $\operatorname{dim}\left(\left|P_{1}^{\prime}\right|\right)=2$. We have $P_{1}^{2}=4+h, K_{S_{1}}^{2}=$ $h-1$, where $h$ is the number of the $(-1)$-cycles on $S$ such that $E \cdot P^{\prime}=0$. Then $\left(P_{1}^{\prime}\right)^{2}=3, P_{1}^{\prime} \cdot K_{S_{1}}=-1$. The analysis of cases (i) and (ii) above tells us that $\left(S_{1},\left|P_{1}^{\prime}\right|\right)$ arises by minimally resolving the base points of either $\left(\mathbb{P}^{2}, \mathcal{L}\left(4 ; 2,1^{9}\right)\right)$ or $\left(\mathbb{P}^{2}, \mathcal{L}\left(6 ; 2^{8}, 1\right)\right)$, and in both cases $h=0$. Note that the cases $\left(\mathbb{P}^{2}, \mathcal{L}\left(5 ; 3,2,1^{9}\right)\right)$ and $\left(\mathbb{P}^{2}, \mathcal{L}\left(6 ; 4,2^{2}, 1^{9}\right)\right)$ are impossible because $k=$ -1 . On the other hand, the pair $\left(\mathbb{P}^{2}, \mathcal{L}\left(6 ; 2^{8}, 1\right)\right)$ does not come by adjunction from a system of curves of genus 2 , whereas the pair $\left(\mathbb{P}^{2}, \mathcal{L}\left(4 ; 2,1^{9}\right)\right)$ does, leading to case (v).

Remark 10.11. Note that in Proposition 10.10(i), the pair $(S, \mathcal{L})$ is also the minimal desingularization of one of the following pairs:

- $\mathcal{L}(4,2)$ for $n=1$

- $\mathcal{L}(5,3,2)$ for $n=0,2$, where for $n=2$ the two base points are infinitely near;

- $\mathcal{L}\left(6 ;\left(4,\left[2^{2}\right]\right)\right)$ for $n=3$.

These linear systems, as well as the others appearing in Proposition 10.10(ii)-(v), are Cremona minimal, because of Corollary 2.4.

In all cases but Proposition 10.10(iii)-(v), one has $h^{1}\left(S, \mathcal{O}_{S}(C)\right)=0$; that is, the system $\mathcal{L}$ is nonspecial. So the existence of these systems is not under question, since one may choose the base points to be general. The question is different for cases (iii)-(v), in which we have $h^{1}\left(S, \mathcal{O}_{S}(C)\right)=1$ in case (iv) and $h^{1}\left(S, \mathcal{O}_{S}(C)\right)=2$ in the remaining two cases. The existence of these systems is discussed in $[\mathrm{DF}]$.

\subsection{Cremona equivalence to smooth curves}

Coolidge [Coo, pages 399-401] considers the question of characterizing those irreducible plane curves $B$ which are Cremona equivalent to a smooth curve. His answers are rather complicated. A simple characterization can be given using Theorem 9.4 (cf. [I3]).

Proposition 10.12. Let $B$ be an irreducible plane curve of genus $g$ which is not Cremona equivalent to a line. Then $B$ is Cremona equivalent to a smooth plane curve of degree $d \equiv 0,1$ modulo 3, if and only if $m(S, C)=[d / 3]$ and, respectively, $\alpha(S, C)=0,1$, where $(S, C)$ is the minimal desingularization of $\left(\mathbb{P}^{2}, B\right)$, and $g=\left(\begin{array}{c}d-1 \\ 2\end{array}\right)$. The same assertion holds for $d \equiv 2$ modulo 3, if $\alpha(S, C)=5$ as soon as either $d \geqslant 29$ or the pair $(S, C)$ does not present the ruled case. 
Proof. We prove only the nontrivial implication. Let $d \equiv 0$ modulo 3 , and assume that $m=m(S, C)=[d / 3]$ and that $\alpha=0$. Then, computing the genera of all pairs in Theorem 9.4 for these values of the invariants, one sees that, in order to have $g=\left(\begin{array}{c}d-1 \\ 2\end{array}\right)$, only case $\left(d p_{1}\right)$ can occur, in which case we have the assertion. The proof of the case $d \equiv 1$ modulo 3 goes in the same way. In the case $d \equiv 2$ modulo 3 , the same proof works as soon as $m>8$. If $m \leqslant 8$, one may have cases in which the genus of the curves in the list of Theorem 9.4 is larger than $\left(\begin{array}{c}d-1 \\ 2\end{array}\right)$ only in the ruled case. The assertion follows.

Coolidge [Coo] also gives conditions under which a plane curve is Cremona equivalent to a curve with only double points. This can be also treated as in Proposition 10.12. We do not dwell on this here.

\section{Appendix A. A proof of the Noether-Castelnuovo theorem via simplicity}

Here we give a proof of the Noether-Castelnuovo theorem (Theorem 1.1) by induction on the simplicity of the homaloidal net $\mathcal{L}$ defining a Cremona transformation $\phi=\phi_{\mathcal{L}}: \mathbb{P}^{2} \rightarrow \mathbb{P}^{2}$; namely, we will show that, if $\phi$ is not quadratic or linear, that is, if its simplicity is larger than $(1,2,0)$, then there is a quadratic transformation $\gamma: \mathbb{P}^{2}--\rightarrow \mathbb{P}^{2}$ such that $\gamma \circ \phi$ is simpler than $\phi$.

The notion of simplicity is essentially due to Alexander [Al1] (cf. the footnote by Segre in [Ca3, page 469] and [Ch]).

Write $\mathcal{L}=\mathcal{L}\left(\delta ; \alpha_{0}, \ldots, \alpha_{r}\right)$, where $\alpha_{0} \geqslant \cdots \geqslant \alpha_{r}$, and let $p_{i}, i=0, \ldots, r$ be the point of multiplicity $\alpha_{i}$ of $\mathcal{L}$. Let $\left(k_{\phi}, h_{\phi}, s_{\phi}\right)$ be the simplicity of $\phi$, which is that of $\mathcal{L}$, as defined in (4). If $\delta=2$, there is nothing to prove. Suppose, then, that $\delta>2$. Note that $k_{\phi}=\delta-\alpha_{0} \geqslant 1$.

By subtracting $\alpha_{0}$ times the latter equation from the former one in (3), one gets

$$
(\delta-1)\left(\delta-3 \alpha_{0}+1\right)=\sum_{i=1}^{r} \alpha_{i}\left(\alpha_{i}-\alpha_{0}\right) \leqslant 0
$$

hence, $\delta<3 \alpha_{0}$ or, equivalently, $\alpha_{0}>k_{\phi} / 2$. Therefore, $h_{\phi} \geqslant 0$. Similarly, by subtracting $m=k_{\phi} / 2$ times the latter equation from the former one in (3), one gets

$$
(\delta-1)(\delta-3 m+1)=\delta(\delta-3 m)+3 m-1=\alpha_{0}\left(\alpha_{0}-m\right)+\sum_{i=1}^{r} \alpha_{i}\left(\alpha_{i}-m\right),
$$


which, since $\delta-3 m=\alpha_{0}-m$ and $m=k_{\phi} / 2 \geqslant 1 / 2$, implies that

$$
\begin{aligned}
2 m\left(\alpha_{0}-m\right) & =\left(\delta-\alpha_{0}\right)\left(\alpha_{0}-m\right)<\sum_{i=1}^{r} \alpha_{i}\left(\alpha_{i}-m\right) \leqslant \sum_{i=1}^{h} \alpha_{i}\left(\alpha_{i}-m\right) \\
& \leqslant \sum_{i=1}^{h} 2 m\left(\alpha_{i}-m\right),
\end{aligned}
$$

where the last inequality follows from the fact that $\alpha_{i} \leqslant \delta-\alpha_{0}=2 m$, for each $i>0$. Thus

$$
\alpha_{0}-m \leqslant \sum_{i=1}^{h}\left(\alpha_{i}-m\right),
$$

which implies that $h_{\phi} \geqslant 2$ and $p_{1}, \ldots, p_{h_{\phi}}$ cannot be all proximate to $p_{0}$; in particular, they cannot be all infinitely near to $p_{0}$ of order 1 . Note that, for $0<i<j \leqslant h_{\phi}$, the points $p_{0}, p_{i}, p_{j}$ are not aligned; otherwise, $\alpha_{0}+\alpha_{i}+\alpha_{j}>$ $\delta$ and $\mathcal{L}$ is reducible, a contradiction.

Suppose first that there are two points among $p_{1}, \ldots, p_{h_{\phi}}$, say $p_{i}$ and $p_{j}$, such that the quadratic transformation $\gamma$ centered at $p_{0}, p_{i}, p_{j}$ exists. Then $\psi=\gamma \circ \phi$ is a Cremona transformation of degree $\delta-\epsilon$, where $\epsilon=\alpha_{i}+\alpha_{j}-$ $2 m>0$, and it is given by a homaloidal net $\Lambda$ having multiplicity $\alpha_{0}-\epsilon<$ $\alpha_{0}, \alpha_{j}-m \leqslant m, \alpha_{i}-m \leqslant m$, respectively, at the points (corresponding to) $p_{0}, p_{i}, p_{j}$. Either $p_{0}$ is still a maximal multiplicity point of $\Lambda$, and so $k_{\psi}=\delta-\alpha_{0}=k_{\phi}$ and $h_{\psi}=h_{\phi}-2$ (because $p_{i}$ and $p_{j}$ are now of multiplicity $\leqslant m=k_{\psi} / 2$ ), or the net $\Lambda$ has maximal multiplicity $\mu>\alpha_{0}-\epsilon$, and so $k_{\psi}<\delta-\alpha_{0}=k_{\phi}$. In both cases, $\psi$ is simpler than $\phi$.

If there is no such quadratic transformation $\gamma$, it means that $p_{i}>p_{0}$, for each $i=1, \ldots, h$, and, by $(21)$, there are $p_{i}, p_{j}$ such that $p_{j}>^{1} p_{i}>^{1} p_{0}$ and $p_{j} \odot p_{0}$. (If $p_{j} \phi p_{0}$, then $\gamma$ exists.) Choosing a general point $q \in \mathbb{P}^{2}$, consider the quadratic transformation $\gamma^{\prime}$ centered at $p_{0}, p_{i}, q$, and set $\psi^{\prime}=\gamma^{\prime} \circ \phi$. Then $\psi^{\prime}$ has degree $\delta+\epsilon^{\prime}$, where $0 \leqslant \epsilon^{\prime}=2 m-\alpha_{i}<m$, and it is given by a homaloidal net $\Lambda^{\prime}$ having multiplicity $\alpha_{0}+\epsilon^{\prime} \geqslant \alpha_{0}, 2 m, \epsilon^{\prime}<m$, respectively, at the points (corresponding to) $p_{0}, p_{i}, q$. Therefore, $k_{\psi^{\prime}}=\delta-\alpha_{0}=k_{\phi}$ and $h_{\psi^{\prime}}=h_{\phi}$. But the base point of $\Lambda^{\prime}$ corresponding to $p_{j}$ is now infinitely near to $p_{0}$ of order 1 ; hence, it is no longer satellite, and $s_{\psi^{\prime}}=s_{\phi}-1$. Therefore, $\psi^{\prime}$ is simpler than $\phi$.

Repeating this argument, we eventually get a Cremona transformation with simplicity $(1,2,0)$, which is a quadratic one, and the proof is concluded. 
Acknowledgment. We warmly thank Professor S. Iitaka for having pointed us to his very recent papers [I3], [I4], [I5], and [I6].

\section{REFERENCES}

[A] M. Alberich-Carramiñana, Geometry of the plane Cremona maps, Lecture Notes in Math. 1769, Springer, Heidelberg, 2002.

[Al1] J. W. Alexander, On factorization of Cremona transformations, Trans. Am. Math. Soc. 17 (1916), 295-300.

[B] L. Bădescu, Algebraic Surfaces, Springer, Berlin, 2001.

[BZ] S. F. Barber and O. Zariski, Reducible exceptional curves of the first kind, Amer. J. Math. 57 (1935), 119-141.

[C] A. Calabri, On rational and ruled double planes, Ann. Mat. Pura Appl. (4) 181 (2002), 365-387.

[Ca1] G. Castelnuovo, Massima dimensione dei sistemi lineari di curve piane di dato genere, Ann. Mat. (2) 18 (1890), 119-128.

[Ca2] G. Castelnuovo, Ricerche generali sopra i sistemi lineari di curve piane, Mem. R. Accad. Sci. Torino Cl. Sci. Fis. Mat. Nat. (2) 42 (1890-1891), 137-188.

[Ca3] G. Castelnuovo, Le trasformazioni generatrici del gruppo cremoniano nel piano, Atti R. Accad. Sci. Torino 36 (1901), 861-874.

[Ch] O. Chisini, Sul teorema di Noether relativo alla decomponibilità di una trasformazione cremoniana in un prodotto di trasformazioni quadratiche, Atti Soc. Nat. Mat. Modena 6 (1921), 7-13.

[CFM] C. Ciliberto, P. Francia, and M. Mendes Lopes, Remarks on the bicanonical map for surfaces of general type, Math. Z. 224 (1997), 137-166.

[Con] F. Conforto, Le superficie razionali, Zanichelli, Bologna, 1939.

[Coo] J. L. Coolidge, A treatise on algebraic plane curves, Dover, New York, 1959.

[DF] M. De Franchis, Riduzione dei fasci di curve piane di genere 2, Rend. Circ. Mat. Palermo 13 (1899), 1-27.

[dP] P. del Pezzo, Sulle superficie di ordine $n$ immerse nello spazio di $n+1$ dimensioni, Rend. R. Acc. Sci. Fis. Mat. Napoli 24 (1885), 212-216.

[D] D. Dicks, Birational pairs according to S. Iitaka, Math. Proc. Cambridge Philos. Soc. 102 (1987), 59-69.

[EH] D. Eisenbud and J. Harris, On varieties of minimal degree (a centennial account), Proc. Sympos. Pure Math. 46 (1987), 3-13.

[EC] F. Enriques and O. Chisini, Lezioni sulla teoria geometrica delle equazioni e delle funzioni algebriche, 4 vols., Zanichelli, Bologna, 1915-1934.

[F1] A. Franchetta, Sulle curve eccezionali riducibili di prima specie, Boll. Unione Mat. Ital. (2) 4 (1940), 332-341.

[F2] A. Franchetta, Sulla caratterizzazione delle curve eccezionali riducibili di prima specie, Boll. Unione Mat. Ital. (2) 5 (1941), 372-375.

[F3] A. Franchetta, Sulle curve riducibili appartenenti ad una superficie algebrica, Rend. Mat. Appl. (5) 8 (1949), 378-398.

[H] R. Hartshorne, Algebraic Geometry, Grad. Texts in Math. 52, Springer, New York, 1977.

[I1] S. Iitaka, On irreducible plane curves, Saitama Math. J. 1 (1983), 47-63.

[I2] S. Iitaka, Birational geometry of plane curves, Tokyo J. Math. 22 (1999), 289-321.

[I3] S. Iitaka, Birational characterization of nonsingular plane curves, preprint, 2006. 
[I4] S. Iitaka, "Birational embedding of algebraic plane curves by mixed pluricanonical maps" in Complex Analysis and Its Applications, OCAMI Stud. 2, Osaka Municipal University Press, Osaka, 2007, 207-211.

[I5] S. Iitaka, "Relationships between $\omega$ and $\sigma$ " in Birational Geometry of Algebraic Plane Curves and Related Topics in Algebraic Geometry, Gakushuin University, Tokyo, 2010, 51-142.

[16] S. Iitaka, "On birational invariants A and $\Omega$ of algebraic plane curves" in Birational Geometry of Algebraic Plane Curves and Related Topics in Algebraic Geometry, Gakushuin University, Tokyo, 2010, 143-164.

[J] G. Jung, Ricerche sui sistemi lineari di curve algebriche di genere qualunque, Ann. Mat. (2) 15 (1888), 277-312; Ricerche sui sistemi lineari di genere qualunque e sulla loro riduzione all'ordine minimo, Ann. Mat. (2) 16 (1889), 291-327.

[K1] S. Kantor, Sur une théorie des courbes et des surfaces admettant des correspondances univoques, C. R. Acad. Sci. Paris Sér. A-B 100 (1885), 343-345.

[K2] S. Kantor, Premiers fondements pour une théorie des transformations périodiques univoques, Atti Accad. Sci. Fis. Mat. Napoli (2) 4 (1891), 1-335.

$[\mathrm{KM}]$ N. M. Kumar and M. P. Murthy, Curves with negative self-intersection on rational surfaces, J. Math. Kyoto Univ. 22 (1982/1983), 767-777.

[M] O. Matsuda, On numerical types of algebraic curves on rational surfaces, Tokyo J. Math. 24 (2001), 359-367.

[Ma] K. Matsuki, Introduction to the Mori program, Springer, New York, 2001.

[MP1] M. Mella and E. Polastri, Equivalent birational embeddings, Bull. Lond. Math. Soc. 41 (2009), 89-93.

[MP2] M. Mella and E. Polastri, Equivalent birational embeddings II: divisors, preprint, arXiv:math/0906.4859 [math.AG].

[Na1] M. Nagata, On rational surfaces, I. Irreducible curves of arithmetic genus 0 or 1 , Mem. Coll. Sci. Univ. Kyoto Ser. A Math. 32 (1960), 351-370.

[Na2] M. Nagata, On rational surfaces, II, Mem. Coll. Sci. Univ. Kyoto Ser. A Math. 33 (1960/1961), 271-293.

[Ne] D. Nencini, Sulla classificazione aritmetica di Noether dei sistemi lineari di curve algebriche piane, Ann. Mat. Pura Appl. (3) 27 (1918), 259-292.

[No] M. Noether, Rationale Ausführung der Operationen in der Theorie der algebraischen Functionen, Math. Ann. 23 (1883), 311-358.

[R] M. Reid, Surfaces of small degree, Math. Ann. 275 (1986), 71-80.

[S] C. Segre, Un'osservazione relativa alla riducibilità delle trasformazioni Cremoniane e dei sistemi lineari di curve piane per mezzo di trasformazioni quadratiche, Atti R. Accad. Sci. Torino 36 (1900-1901), 645-651.

\author{
Alberto Calabri \\ Dipartimento di Matematica \\ Università degli Studi di Ferrara \\ 44121 Ferrara \\ Italy \\ alberto.calabri@unife.it
}


Ciro Ciliberto

Dipartimento di Matematica

Università degli Studi di Roma "Tor Vergata"

00133 Roma

Italy

cilibert@mat.uniroma2.it 